\title{
Probing mild-tempered neutralino dark matter through top-squark production at the LHC
}

\author{
Monoranjan Guchait, ${ }^{1, *}$ Arnab Roy, ${ }^{1, \dagger}$ and Seema Sharma ${ }^{2, \$}$ \\ ${ }^{1}$ Department of High Energy Physics, Tata Institute of Fundamental Research, \\ Homi Bhabha Road, Mumbai 400005, India \\ ${ }^{2}$ Indian Institute of Science Education and Research, Pune 411008, India
}

(Received 29 June 2021; accepted 3 September 2021; published 23 September 2021)

\begin{abstract}
The lightest neutralino, assumed to be the lightest supersymmetric particle, is proposed to be a dark matter $(\mathrm{DM})$ candidate for the mass $\mathcal{O}(100) \mathrm{GeV}$. Constraints from various direct dark matter detection experiments and Planck measurements exclude a substantial region of parameter space of minimal supersymmetric standard model. However, a "mild-tempered" neutralino with dominant bino composition and a little admixture of Higgsino is found to be a viable candidate for DM. Within the minimal supersymmetric standard model framework, we revisit the allowed region of parameter space that is consistent with all existing constraints. Regions of parameters that are not sensitive to direct detection experiments, known as "blind spots," are also revisited. Complimentary to the direct detection of DM particles, a mild-tempered neutralino scenario is explored at the LHC with the center of mass energy $\sqrt{\mathrm{s}}=13 \mathrm{TeV}$ through the top-squark pair production, and its subsequent decays with the standard-modellike Higgs boson in the final state. Our considered channel is found to be very sensitive also to the blind spot scenario. Detectable signal sensitivities are achieved using the cut-based method for the high luminosity options 300 and $3000 \mathrm{fb}^{-1}$, which are further improved by applying the multivariate analysis technique.
\end{abstract}

DOI: $10.1103 /$ PhysRevD.104.055032

\section{INTRODUCTION}

The quest for a signature of beyond standard model (SM) physics is a very high priority agenda in high energy physics experiments and it has been going on for a long time in several laboratories. In particular, at the LHC experiments, looking for new physics signals is the major thrust area. Unfortunately, no single direct evidence of new physics signals has been observed at this point. As a consequence, the absence of experimental confirmation leads to stringent constraints to various beyond SMs [1]. On the other hand, the well-confirmed existence of dark matter (DM) by various cosmological and astrophysical experiments serves as one of the strong motivations to propose the existence of beyond SM physics [2,3]. Among several probable candidates of DM, the weakly interacting massive particle (WIMP) turns out to be the most suitable one for

\footnotetext{
*guchait@tifr.res.in †arnab.roy@tifr.res.in

"seema@iiserpune.ac.in
}

Published by the American Physical Society under the terms of the Creative Commons Attribution 4.0 International license. Further distribution of this work must maintain attribution to the author(s) and the published article's title, journal citation, and DOI. Funded by SCOAP. thermal DM, with a correct relic density measured by a PLANCK experiment, which predicts [4]

$$
\Omega \mathrm{h}^{2}=0.12 \pm 0.001
$$

Enormous efforts have been in place for a long time to look for DM candidates via direct and indirect searches in various experiments [5-10]. However, null results, in particular, from some of the direct detection (DD) experiments have resulted in strong constraints on DM-nucleon scattering cross sections in terms of DM (WIMP) masses [11-20]. The DM-nucleon scattering cross section can be classified into two categories, namely, spin independent (SI) and spin dependent (SD), depending on the structure of the coupling. Note that, in general, the SI DM-nucleon scattering cross section is smaller than that of the SD case, and it is more sensitive to DD experiments [21-23]. For instance, the most stringent bounds come from XENON1T experiment, where the DM-nucleon scattering cross section corresponding to the DM of the mass range $\sim 20-100 \mathrm{GeV}$ is strongly restricted, $\sigma_{\mathrm{SI}} \lesssim 10^{-46} \mathrm{~cm}^{2}$ [13]. The other experiments, such as LUX [11], PANDA [12], PICO-60 [17], Darkside [14] etc., also constrain the DM-nucleon cross section for a wide range of masses of DM candidates from few $\mathrm{GeV}$ to $\mathrm{TeV}$. 
The minimal supersymmetric standard model (MSSM) with R-parity conservation offers the lightest neutralino, assumed to be the lightest supersymmetric particle (LSP) as the potential DM (WIMP) candidate of the mass $\sim 100 \mathrm{GeV}$ [24-27]. Comprehensive searches of neutralino DM are carried out at the LHC, which lead to various constraints in the absence of any signal [28-30]. In MSSM, the physical neutralino state is constituted through the linear superposition of electroweak gauginos [bino ( $\tilde{\mathrm{B}})$, wino $(\tilde{\mathrm{W}})]$ and Higgsinos $\left(\tilde{\mathrm{H}}_{\mathrm{u}}^{0}, \tilde{\mathrm{H}}_{\mathrm{d}}^{0}\right)$. This composition is mainly determined by relative values of two electroweak gaugino mass parameters, $\mathrm{M}_{1}$ and $\mathrm{M}_{2}$, corresponding to $\mathrm{U}(1)$ and $\mathrm{SU}(2)$ gauge transformations, respectively. In addition, the other two parameters, namely Higgsino mass parameter $(\mu)$ and $\tan \beta$, and the ratio of two vacuum expectation values of two neutral Higgs bosons also play an important role in determining the physical masses and composition of neutralino states. A neutralino state with pure Higgsino or wino composition of the mass $\sim \mathcal{O}(100) \mathrm{GeV}$ is found not to be a favorable DM candidate because of its underabundance of relic density [31]. However, for large masses $\mathcal{O}(\mathrm{TeV})$, those can serve as a DM candidate [31-35]. Similarly, a neutralino with pure bino composition also does not satisfy the right relic density measurement [see Eq. (1.1)]. Hence, in order to propose LSP as a viable DM candidate, the "tempered neutralino" scenario is proposed to be the best bet [31], where the neutralino is no longer a pure state but has admixtures of more than one composition. A well-tempered bino-Higgsino [36-39] or bino-wino [40-42] neutralino is found to be the most suitable DM candidate for the mass $\sim 100 \mathrm{GeV}$ to achieve the right relic density. The Higgsino component is indispensable to bring down the relic density to the required value [Eq. (1.1)] via resonant $\mathrm{Z}$ or Higgsmediated annihilation, where the Higgs can be a SM-like Higgs boson as well as heavier Higgs boson states in the limit of large sfermion masses. It is to be noted that the neutralino-nucleon SI scattering cross section is enhanced with the increase of Higgsino composition in the neutralino state. Therefore, the strong experimental limits on the SI scattering cross section restrict the composition of neutralinos, in particular, the Higgsino content $[43,44]$. Hence a bino-dominated neutralino with a little mixture of Higgsino component, referred to as "mild-tempered neutralino," is expected to be the viable DM candidate for the mass $\mathcal{O}(100) \mathrm{GeV}$ or little less [45], and consistent with all existing constraints. In this regard, it is to be noted that few studies exist in the literature based on the extended supersymmetric (SUSY) model, which present very light DM candidates $\left(\mathrm{m}_{\chi} \lesssim 50 \mathrm{GeV}\right)$ [46-50], satisfying all current constraints.

It is worth pointing out here that there exists a region of MSSM parameter space where the DM-nucleon SI scattering cross section almost vanishes because of the interplay among various amplitudes. Consequently, direct detection rate of DM becomes insensitive corresponding to that region of parameter space, which is known as the "blind spot" (BS) [51-55]. As the DD experiments fail to probe this BS scenario, it is worth finding a complementary way for DM searches at the LHC.

In this current study, we focus on the mild-tempered scenario, i.e., bino-Higgsino neutralino with a larger bino component, and of the mass $\mathcal{O}(100) \mathrm{GeV}$, and then identify the corresponding region of parameter space consistent with all measurements. The existence of a relatively lighter LSP of the mass range considered in this study is still not absolutely ruled out by any SUSY searches at the LHC. Hence, our study will presumably give some idea about its detectability at the LHC with its high luminosity options. With this aim, the characteristic signature corresponding to this mildtempered neutralino including the BS scenario are discussed for the LHC experiment. We consider the top-squark pair production and then its cascade decay to SM-like Higgs boson and an LSP, the DM candidate. Although $\mathrm{m}_{\tilde{t}_{1}} \lesssim$ 1.1 TeV are ruled out from searches at the LHC in the context of various simplified models, for low $\operatorname{BR}\left(\tilde{t}_{1} \rightarrow \tilde{\chi}_{1}^{0}+\mathrm{t}\right) \sim 10 \%, \mathrm{~m}_{\tilde{t}_{1}}<1 \mathrm{TeV}$ are found to be still allowed using statistical analysis. It is be noted that the top squarks of lower mass range, which are within the reach of current LHC energy, are also motivated in the context of "naturalness" scenario [56-61]. A detailed investigation is carried out performing simulation to explore the feasibility of finding the signal at the LHC for higher luminosity options, such as $\mathcal{L}=300 \mathrm{fb}^{-1}$ and $3000 \mathrm{fb}^{-1}$.

The paper is organized as follows. In Sec. II, the MSSM model set up providing mild-tempered neutralino and BS scenario is discussed, and then corresponding allowed region of parameters are identified. In Sec. III, signal and background simulations are presented and followed by results. Finally, we summarize in Sec. IV.

\section{MILD-TEMPERED NEUTRALINO SCENARIO IN THE MSSM}

In this section, we discuss the MSSM model setup and then delineate the region of parameter space interesting to our scenario which presents a DM candidate of mass $\sim \mathcal{O}(100) \mathrm{GeV}$ consistent with the existing data from Planck experiment [Eq. (1.1)] and direct searches as mentioned above.

In the gauge eigenstate basis $\left(\tilde{\mathrm{B}}, \tilde{\mathrm{W}}_{3}, \tilde{\mathrm{H}}_{\mathrm{d}}^{0}, \tilde{\mathrm{H}}_{\mathrm{u}}^{0}\right)$, the neutralino mass matrix can be written as

$$
\mathbf{M}_{\mathrm{N}}=\left(\begin{array}{cccc}
\mathbf{M}_{1} & 0 & \frac{-g_{1} v c_{\beta}}{\sqrt{2}} & \frac{g_{1} v s_{\beta}}{\sqrt{2}} \\
0 & \mathbf{M}_{2} & \frac{g_{2} v c_{\beta}}{\sqrt{2}} & \frac{-g_{2} v s_{\beta}}{\sqrt{2}} \\
\frac{-g_{1} v c_{\beta}}{\sqrt{2}} & \frac{g_{2} v c_{\beta}}{\sqrt{2}} & 0 & -\mu \\
\frac{g_{1} v s_{\beta}}{\sqrt{2}} & \frac{-g_{2} v s_{\beta}}{\sqrt{2}} & -\mu & 0
\end{array}\right) .
$$


Here, $\mathrm{M}_{1}\left(\mathrm{~g}_{1}\right)$ and $\mathrm{M}_{2}\left(\mathrm{~g}_{2}\right)$ present the $(\mathrm{U}(1)) \tilde{\mathrm{B}}$ and (SU (2)) $\tilde{\mathrm{W}}_{3}$ gaugino mass (coupling) parameters, respectively, whereas $\mu$ is defined to be the Higgsino mass parameter. The two vacuum expectation values corresponding to two neutral components of the two Higgs doublets $\mathrm{H}_{\mathrm{u}}^{0}$ and $\mathrm{H}_{\mathrm{d}}^{0}$ are $v_{u}$ and $v_{d}$, respectively and constrained to be $v_{u}^{2}+v_{d}^{2}=v^{2}$. As practice, we assume $\tan \beta=\frac{v_{u}}{v_{d}}$, and $\mathrm{s}_{\beta} \equiv \sin \beta, \mathrm{c}_{\beta} \equiv \cos \beta$. The symmetric matrix $\mathrm{M}_{\mathrm{N}}$ can be diagonalized by a unitary matrix $\mathrm{N}_{4 \times 4}$ to obtain the masses of four neutralino states $\tilde{\chi}_{\mathrm{i}}^{0}(\mathrm{i}=1,2,3,4)$ as

$$
\mathrm{M}_{\tilde{\chi}^{0}}^{\mathrm{D}}=\mathrm{NM}_{\mathrm{N}} \mathrm{N}^{\dagger}
$$

and the corresponding physical neutralino states are given by

$$
\tilde{\chi}_{\mathrm{i}}^{0}=\mathrm{N}_{\mathrm{i} 1} \tilde{\mathrm{B}}+\mathrm{N}_{\mathrm{i} 2} \tilde{\mathrm{W}}_{3}+\mathrm{N}_{\mathrm{i} 3} \tilde{\mathrm{H}}_{\mathrm{d}}^{0}+\mathrm{N}_{\mathrm{i} 4} \tilde{\mathrm{H}}_{\mathrm{u}}^{0} .
$$

Among the four neutralino states, two of the lighter states become gaugino-like ( $\tilde{\mathrm{B}}$ and $\tilde{W}_{3}$ ), if $\left|\mathrm{M}_{1,2}-\mu\right| \geq \mathrm{M}_{\mathrm{Z}}$ and $|\mu|>\mathrm{M}_{2}>\mathrm{M}_{1}$ with masses $\mathrm{m}_{\tilde{\chi}_{1}^{0}} \sim \mathrm{M}_{1}$ and $\mathrm{m}_{\tilde{\chi}_{2}^{0}} \sim \mathrm{M}_{2}$, respectively. The masses of Higgsino-dominated states are mostly controlled by $\mu$, and in particular $\mathrm{m}_{\tilde{\chi}_{2,3}^{0}} \sim \mu$ for a decoupled scenario $\left(\mathrm{M}_{2} \gg \mu>\mathrm{M}_{1}\right)$. Further, for $\mathrm{M}_{1}<\mu \ll \mathrm{M}_{2}$ cases, the heaviest state is expected to be $\sim \tilde{\mathrm{W}}_{3}$-like, whereas intermediate states become Higgsinodominated with the lightest state almost bino-like with tiny Higgsino component (i.e., $\mathrm{N}_{11}^{2} \gg \mathrm{N}_{13}^{2}+\mathrm{N}_{14}^{2}$ ). Similarly, in the basis $\left(\mathrm{iW}^{-}, \tilde{\mathrm{H}}_{\mathrm{u}}^{-}\right)$and $\left(\mathrm{i} \tilde{\mathrm{W}}^{+}, \tilde{\mathrm{H}}_{\mathrm{d}}^{+}\right)$the chargino mass matrix is given by

$$
\mathrm{M}_{\mathrm{C}}=\left(\begin{array}{cc}
\mathrm{M}_{2} & \sqrt{2} \mathrm{M}_{\mathrm{W}} \sin \beta \\
\sqrt{2} \mathrm{M}_{\mathrm{W}} \cos \beta & \mu
\end{array}\right),
$$

which is diagonalized by two unitary matrices $U$ and $V$. For $\mathbf{M}_{2} \gg \mu$, the lighter chargino $\left(\tilde{\chi}_{1}^{ \pm}\right)$state becomes Higgsino-like.

For our considered scenario, the dominant DM annihilation process occurs through the $s$ channel mediated by $C P$-even $(\mathrm{h}, \mathrm{H})$ and $C P$-odd (A) Higgs bosons or $\mathrm{Z}$,

$$
\tilde{\chi}_{1}^{0} \tilde{\chi}_{1}^{0} \stackrel{\phi / \mathrm{Z}}{\rightarrow} \mathrm{ff}, \quad \phi=\mathrm{h}, \mathrm{H}, \mathrm{A},
$$

in the limit of relatively heavier slepton masses. The cross section of the annihilation process primarily depends on the $(\phi, Z)-\tilde{\chi}_{1}^{0}-\tilde{\chi}_{1}^{0}$ couplings, which are of the following form:

$$
\begin{aligned}
& \mathrm{g}_{\mathrm{h} \tilde{\chi}_{1}^{0} \tilde{\chi}_{1}^{0}} \sim \mathrm{g}\left(\mathrm{N}_{12}-\tan \theta_{\mathrm{W}} \mathrm{N}_{11}\right)\left(\sin \alpha \mathrm{N}_{13}+\cos \alpha \mathrm{N}_{14}\right), \\
& \mathrm{g}_{\mathrm{H} \tilde{\chi}_{1}^{0} \tilde{\chi}_{1}^{0}} \sim \mathrm{g}\left(\mathrm{N}_{12}-\tan \theta_{\mathrm{W}_{11}} \mathrm{~N}_{11}\right)\left(\sin \alpha \mathrm{N}_{14}-\cos \alpha \mathrm{N}_{13}\right), \\
& \mathrm{g}_{\mathrm{A} \tilde{\chi}_{1}^{0} \tilde{\chi}_{1}^{0}} \sim \mathrm{g}\left(\mathrm{N}_{12}-\tan \theta_{\mathrm{W}} \mathrm{N}_{11}\right)\left(\cos \beta \mathrm{N}_{14}-\sin \beta \mathrm{N}_{13}\right),
\end{aligned}
$$

$$
\mathrm{g}_{\mathrm{Z} \tilde{\chi}_{1}^{0} \tilde{x}_{1}^{0}} \sim \frac{\mathrm{g}}{2 \cos \theta_{\mathrm{W}}}\left(\mathrm{N}_{13}^{2}-\mathrm{N}_{14}^{2}\right),
$$

where $\alpha$ is the mixing angle of the $C P$-even Higgs sector. Clearly, the combined effect of bino $\left(\mathrm{N}_{11}\right)$ and Higgsino components $\left(\mathrm{N}_{13}, \mathrm{~N}_{14}\right)$ in $\tilde{\chi}_{1}^{0}$ determine the annihilation rate. In addition, a neutralino with a moderate to large amount of Higgsino content may dominantly coannihilate with Higgsino-like (large $\mathrm{V}_{12}$ ) and nearly mass degenerate lighter charginos $\tilde{\chi}_{1}^{ \pm}$,

$$
\tilde{\chi}_{1}^{0} \tilde{\chi}_{1}^{ \pm} \stackrel{\mathrm{W}^{ \pm}}{\rightarrow} \mathrm{f} \overline{\mathrm{f}},
$$

along with other subdominant contributions, which may enhance the annihilation cross section through the following coupling:

$$
\mathrm{g}_{\mathrm{W}^{ \pm} \tilde{\chi}_{1}^{0} \tilde{\chi}_{1}^{ \pm}}=\frac{\mathrm{g} \tan \theta_{\mathrm{W}}}{\sqrt{2}}\left(\mathrm{~N}_{14} \mathrm{~V}_{12}^{*}-\sqrt{2} \mathrm{~N}_{12} \mathrm{~V}_{11}^{*}\right) .
$$

Hence, in combination of all processes, whichever are viable, the cross section for annihilation process corresponding to a Higgsino-like LSP goes up leading to an underabundance of relic density. Hence one can conclude that an LSP with a suitable combination of bino and Higgsino composition appears to be a viable DM candidate around the mass $\mathcal{O}(100) \mathrm{GeV}$. In the case of winoHiggsino-dominated LSP, various possible annihilation and coannihilation processes can occur, which are mediated by SM gauge bosons and lead to the underabundant scenario. In order to achieve the right relic density prediction, in this case, one needs to lift the mass $\left(\sim \mathrm{M}_{2}\right)$ of the LSP to TeV level and suppress annihilation cross section [31,32]. This type of scenario appears naturally in anomaly mediated SUSY breaking model [62-64]. Hence, a SUSY DM model disfavors the possibility of Higgsino-/ wino-dominated scenario with DM mass $\sim \mathcal{O}(100) \mathrm{GeV}$.

As pointed out earlier, the composition of the LSP DM candidate is also constrained by direct detection experiments [11-13,16,17], where DM candidate scattering off a heavy nucleus mediated by Higgs/gauge bosons or squarks. The effects due to heavier squarks $[\mathcal{O}(1) \mathrm{TeV}]$ are very much suppressed. Hence the main contribution to SI (SD) cross section occurs through Higgs (Z) boson exchange via $t$-channel diagram $[21,22]$. The dominant contribution comes from the diagram mediated by the $C P$-even lightest Higgs boson, whereas contributions due to other heavier Higgs bosons are suppressed. Interestingly, this suppression can be compensated by enhanced couplings of heavier Higgs bosons with the quarks for a certain range of parameters, in particular, for higher values of $\tan \beta$, which we will discuss later.

The SI scattering cross section is also sensitive to couplings $\mathrm{g}_{\mathrm{h} \tilde{\chi}_{1}^{0} \tilde{\chi}_{1}^{0}}$ [Eq. (2.6)]. The presence of a larger Higgsino component in $\tilde{\chi}_{1}^{0}$ enhances the SI DM-nucleon 

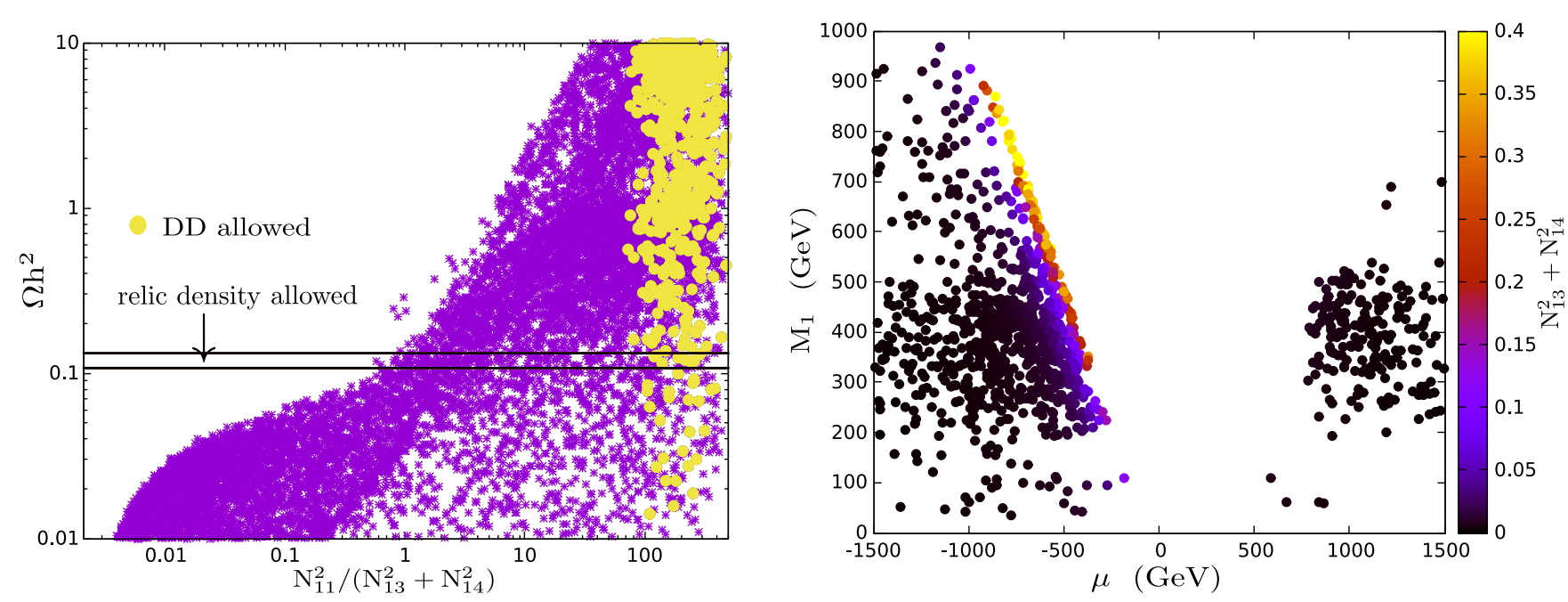

FIG. 1. Left: relic density with the variation of $\tilde{\mathrm{B}} / \tilde{\mathrm{H}}$ components in $\tilde{\chi}_{1}^{0}$. Allowed points by DD measurements (yellow) and relic density (black band). Right: ranges of $\mu$ and $\mathrm{M}_{1}$ along with the Higgsino component of the LSP allowed by relic density and DD constraints.

scattering cross section mediated mainly by the $C P$-even lightest Higgs boson, which is tightly constrained by the existing limits on DM-nucleon scattering cross section from the XENON1T experiment [13]. On the other hand, the composition of the $\tilde{\chi}_{1}^{0}$ state is also constrained by relic density. Hence, the bino-Higgsino content of a mildtempered neutralino state is severely restricted by the combined effect of relic density measurements and DMnucleon cross section limits. This feature of mild-tempered neutralino is reflected in Fig. 1 (left), where the variation of relic density with the relative size of bino and Higgsino compositions of the LSP are presented in terms of $\mathrm{N}_{11}^{2} /\left(\mathrm{N}_{13}^{2}+\mathrm{N}_{14}^{2}\right)$. It is to be noted that this figure is subject to the condition $\mu>0$ to avoid effects from "blind spots," which occur for $\mu<0$ when $\mathrm{M}_{1}$ is assumed to be positive. It will be discussed in detail later. In Fig. 1 (right), we present the ranges of $\mu$ and $\mathrm{M}_{1}$ allowed by relic density, limits from DD experiments along with some other constraints as described in Sec. II A. These figures are obtained by performing a numerical scan of parameters [Eq. (2.17)], which will be discussed later.

Figure 1 (left) indicates that the $\tilde{\chi}_{1}^{0}$, with a relatively higher Higgsino composition and tiny bino content, makes underabundance of relic density. In this case, along with DM annihilation [Eq. (2.5)], the coannihilation process [Eq. (2.10)] also takes place resulting in a larger DM annihilation cross section. Towards the rightmost region of Fig. 1 (left), due to the absence of sufficient Higgsino components in the LSP, the DM-nucleon scattering cross section goes down because of the couplings [Eq. (2.6)] and becomes consistent with DD limits. This region is presented (yellow) in Fig. 1 (left) at the higher values of ratio $\mathrm{N}_{11}^{2} /\left(\mathrm{N}_{13}^{2}+\mathrm{N}_{14}^{2}\right)$. Hence, it can be concluded that the binodominated LSP with little admixtures $(\sim 1 \%)$ of Higgsino component is the most favoured option in a decoupled scenario $\left(\mathrm{M}_{2}\right.$ is very large $)$. We referred to this as a scenario of "mild-tempered" neutralino in the previous section. In this scenario, relatively higher values $\mu$ are found to be allowed with light to moderate values of $\mathrm{M}_{1}$. It is clearly seen in Fig. 1 (right) that, for $\mu>0$, the Higgsino fraction in the LSP is tiny. However, for $\mu<0$, there exist parameter spaces with a comparatively higher amount of Higgsino components that are still allowed. It occurs mainly due to the effect of "blind spots," which is discussed next.

A blind spot is an interesting scenario where the DMnucleon scattering cross section is found to be very insensitive for a certain range of relevant parameters in the MSSM, and the corresponding region of parameters is called a "blind spot." It may happen for various reasons. For instance, the tree-level scattering cross section may vanish either for a pure gaugino (i.e., $\mathrm{N}_{13}, \mathrm{~N}_{14} \sim 0$ ) or Higgsino (i.e., $\mathrm{N}_{11} \sim 0$ ) neutralino state. Moreover, scattering takes place via one and two loop diagrams mediated by gauge bosons, and an accidental cancellation among various scattering amplitudes for pure Higgsino and gaugino LSP states makes the total cross sections too small and beyond the sensitivity of DD experiment $\left(\sigma_{\mathrm{SI}} \ll 10^{-46} \mathrm{~cm}^{2}\right)[51,65,66]$. Finally, BS may also arise at the tree level due to cancellation among various amplitudes. The dominant contribution to DM-nucleon cross section comes from the diagram mediated by the $C P$-even lightest Higgs boson, whereas contributions due to other heavier Higgs bosons are found to be very small for the decoupling scenario $\left(\mathrm{m}_{\mathrm{A}} \gg \mathrm{M}_{\mathrm{Z}}\right)$. Interestingly, at the tree level, the suppression of contribution mediated by heavier Higgs bosons can be compensated by its enhanced coupling with the (down type) fermions for the range of moderate to higher values of $\tan \beta$. Additionally, the coupling between heavier Higgs bosons and neutralinos, $\mathrm{H}-\tilde{\chi}_{1}^{0}-\tilde{\chi}_{1}^{0}$ [Eq. (2.7)] may receive similar kind of enhancement for a larger value of $\mathrm{N}_{13}$, the down type of Higgsino content in the LSP. 
Consequently, the amplitudes mediated by heavier Higgs bosons turn out to be comparable or at the same level of the $C P$-even SM-like Higgs boson exchange diagram. Depending on the relative signs of $\mu$ and $\mathrm{M}_{1}$, the interference between these two diagrams may become destructive or constructive [53]. Incidentally, for a certain range and combination of related parameters, these two contributions almost cancel each other leading to a scattering insensitive cross section [53]. A detailed analytical study shows that the combination of parameters corresponding to the BS for moderate to larger values of $\tan \beta$ follow the relation among $\mu, \mathrm{m}_{\mathrm{A}}, \tan \beta$, and $\mathrm{m}_{\tilde{\chi}_{1}^{0}}\left(\sim \mathrm{M}_{1}\right)$, as [53]

$$
\frac{\mathrm{M}_{1}}{\mu} \sim-\left(\sin 2 \beta+\tan \beta \frac{\mathrm{m}_{\mathrm{h}}^{2}}{2 \mathrm{~m}_{\mathrm{A}}^{2}}\right) .
$$

Corresponding to this parameter space, naturally a larger Higgsino component $\sim \mathcal{O}(10 \%)$ can be accessible without violating $\mathrm{DD}$ bounds in contrast to the requirement of $\sim \mathcal{O}(1 \%)$ or less for a mild-tempered neutralino case. The above condition for BS connects the gaugino mass parameter with the Higgs sector.

Note that a substantial region of $\tan \beta$ and $\mathrm{m}_{\mathrm{A}}$ plane is excluded from the Higgs searches in the channel, h, A $\rightarrow \tau \tau$ $[67,68]$. This $\mathrm{m}_{\mathrm{A}}-\tan \beta$ exclusion can be traded to obtain constraints on $\mu / \mathrm{m}_{\tilde{\chi}_{1}^{0}}$ by using Eq. (2.12) in the $\mathrm{m}_{\mathrm{A}^{-}}$- $\tan \beta$ plane. In Fig. 2, following Eq. (2.12), the contour plots of $\frac{\mu}{m_{\tilde{\chi}_{1}^{0}}} \sim \frac{\mu}{\mathrm{M}_{1}}$ are shown in the $\mathrm{m}_{\mathrm{A}}, \tan \beta$ plane [53]. The region above the red and blue lines are excluded due to the nonobservation of any signal events in the $\mathrm{h}, \mathrm{A} \rightarrow \tau \tau$

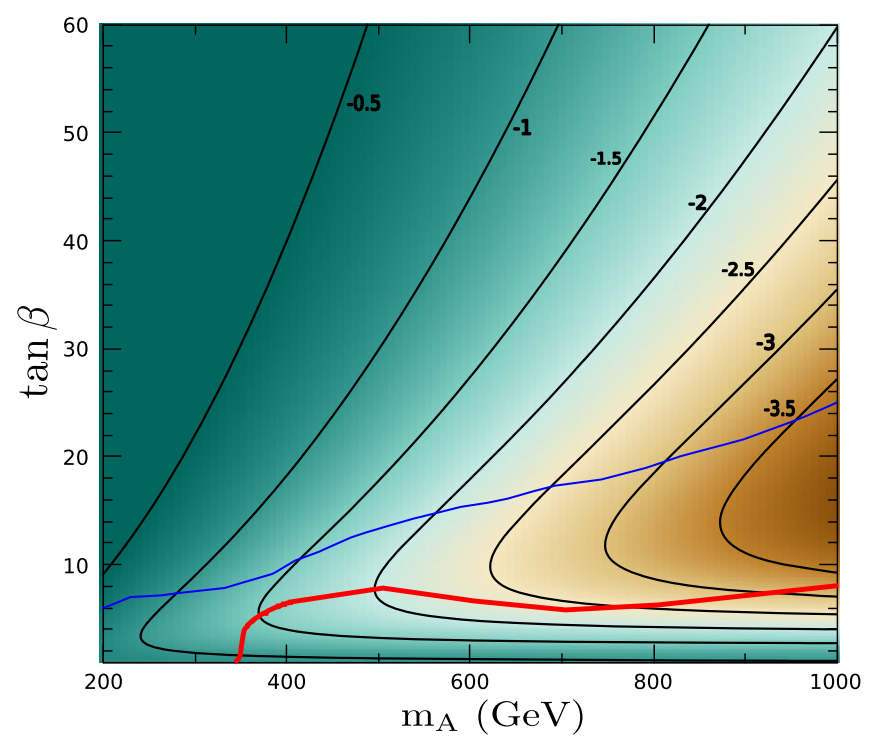

FIG. 2. Contour plots of $\mu / \mathrm{m}_{\tilde{\chi}_{1}^{0}}$ for various values corresponding to a BS scenario in the $\tan \beta$ and $\mathrm{m}_{\mathrm{A}}$ plane along with the exclusion lines from $\mathrm{h}, \mathrm{A} \rightarrow \tau \tau$ searches in ATLAS (red) and CMS (blue) experiments. searches by ATLAS [67] $\left(\mathcal{L}=139.5 \mathrm{fb}^{-1}\right)$ and CMS [68] $\left(\mathcal{L}=35.9 \mathrm{fb}^{-1}\right)$ experiments, respectively. Depending on the value of $\mathrm{m}_{\mathrm{A}}$, the $\mathrm{BS}$ condition, i.e., the ratio $\frac{\mu}{\mathrm{M}_{1}}$ may vary from -1.5 to -3.5 . It implies that the lightest neutralino state is bino-like, whereas the second and third heavier states are Higgsino-like in the limit of large $\mathrm{M}_{2}$, which is exactly the scenario that we try to explore at the LHC experiment.

As explained before, the main focus of this study is to explore the feasibility of finding a mild-tempered neutralino scenario at the LHC. The added advantage of our proposed channel is its sensitiveness to the region of parameters corresponding to the BS scenario, which can also be probed at the LHC. As we know, the content of bino and Higgsino in the LSP depends on the splitting between $\mu$ and $\mathrm{M}_{1}$. Therefore, a mild-tempered scenario appears with the condition $|\mu|-\mathrm{M}_{1} \gtrsim 100 \mathrm{GeV}$, which provides also an LSP of mass $\mathcal{O}(100) \mathrm{GeV}$. The scenario of a little Higgsino admixture along with the dominant bino composition in $\tilde{\chi}_{1}^{0}$ presumably predicts Higgsino-dominated $\tilde{\chi}_{2,3}^{0}$ and $\tilde{\chi}_{1}^{ \pm}$states that are degenerate in mass $\sim \mu$ for a decoupled wino state (i.e., large $\mathrm{M}_{2}$ ). In such cases, heavier states $\tilde{\chi}_{2,3}^{0}$ prefer to decay to a $\mathrm{Z}$ boson and an LSP, and $\tilde{\chi}_{1}^{ \pm}$decays to a W and an LSP. The coupling involved in $\tilde{\chi}_{2,3}^{0}$ decays is $\mathrm{Z}-\tilde{\chi}_{2,3}^{0}-\tilde{\chi}_{1}^{0} \propto \mathrm{N}_{13} \mathrm{~N}_{23}-\mathrm{N}_{14} \mathrm{~N}_{24}$, and since $\tilde{\chi}_{1}^{0}$ is primarily bino dominated (i.e., $\mathrm{N}_{13}, \mathrm{~N}_{14}$ is very tiny), it is suppressed. Thus $\tilde{\chi}_{2,3}^{0}$ preferably decay as

$$
\tilde{\chi}_{2,3}^{0} \rightarrow \mathrm{h}+\tilde{\chi}_{1}^{0},
$$

and a larger Higgsino composition in $\tilde{\chi}_{2,3}^{0}$ state [Eq. (2.6)] makes its rate higher. This decay channel of $\tilde{\chi}_{2,3}^{0}$ is found to be the characteristic feature for the mild-tempered neutralino scenario. Hence testing of this scenario can be performed by studying $\tilde{\chi}_{2,3}^{0}$ and $\tilde{\chi}_{1}^{ \pm}$production at the LHC $[69,70]$ and their subsequent decays. Earlier, this channel is thought to be a "spoiler" mode corresponding to trilepton signal in $\mathrm{pp} \rightarrow$ $\tilde{\chi}_{2}^{0} \tilde{\chi}_{1}^{ \pm} \rightarrow \ell^{+} \ell^{-} \ell \tilde{\chi}_{1}^{0} \tilde{\chi}_{1}^{0}$ production $[71,72]$. In this study, instead of considering the $\tilde{\chi}_{2}^{0} \tilde{\chi}_{1}^{ \pm}$production via electroweak interaction, we consider the production of $\tilde{\chi}_{2}^{0}$ through lighter top-squark production via strong interaction, where $\tilde{\mathrm{t}_{1}}$ dominantly decays to Higgsino-like $\tilde{\chi}_{2,3}^{0}$ and $\chi_{1}^{ \pm}[70,73]$. The decay $\tilde{\mathrm{t}}_{1} \rightarrow \mathrm{t}+\tilde{\chi}_{2,3}^{0}$, is governed by the interactions

$$
\mathcal{L}_{\overline{\mathrm{t}} \tilde{\chi}_{\mathrm{i}}^{0}}=\overline{\mathfrak{t}}\left(\mathrm{g}_{\mathrm{L}}^{\tilde{\chi}_{\mathrm{i}}^{0}} \mathrm{P}_{\mathrm{L}}+\mathrm{g}_{\mathrm{R}}^{\tilde{\chi}_{\mathrm{i}}^{0}} \mathrm{P}_{\mathrm{R}}\right) \tilde{\chi}_{\mathrm{i}}^{0} \tilde{\mathrm{t}}
$$

where

$$
\begin{aligned}
\tilde{g}_{L}^{0} & =-\left[\frac{g_{2}}{\sqrt{2}} N_{i 2}+\frac{g_{1}}{3 \sqrt{2}} N_{i 1}\right] \cos \theta_{\tilde{t}}-\frac{m_{t}}{v} N_{i 4} \sin \theta_{\tilde{t}} \\
& \sim \frac{g_{1}}{3 \sqrt{2}} N_{i 1} \cos \theta_{\tilde{t}}-\frac{m_{t}}{v} N_{i 4} \sin \theta_{\tilde{t}},
\end{aligned}
$$




$$
g_{R}^{\tilde{\chi}_{i}^{0}}=\frac{2 \sqrt{2}}{3} g_{1} N_{i 1} \sin \theta_{\tilde{t}}-\frac{m_{t}}{v} N_{i 4} \cos \theta_{\tilde{t}} .
$$

Here $\theta_{\tilde{t}}$ is the mixing angle in the top-squark sector. Evidently, the $\mathrm{m}_{\mathrm{t}}$ dependent term becomes dominant for Higgsino $\left(\mathrm{N}_{\mathrm{i} 4}\right)$ like neutralino states leading higher branching ratio (BR) for $\tilde{\mathrm{t}_{1}} \rightarrow \tilde{\chi}_{2,3}^{0}+\mathrm{t}$. Due to large enough splitting between $\mu$ and $\mathrm{M}_{1}$, it is natural to have $\mathrm{m}_{\tilde{\chi}_{2,3}^{0}}-\mathrm{m}_{\tilde{\chi}_{1}^{0}}>125 \mathrm{GeV}$, resulting in the $\tilde{\chi}_{2,3}^{0} \rightarrow \mathrm{h}+\tilde{\chi}_{1}^{0}$ decay to be dominant. Hence the mildtempered neutralino DM can be indirectly produced from the decay of Higgsino-like $\tilde{\chi}_{2,3}^{0}$ producing those in the lighter topsquark $\left(\tilde{t_{1}}\right)$ production. It is be noted that this type of scenario can also be probed through the associated production, such as $\mathrm{pp} \rightarrow \tilde{\chi}_{2,3}^{0} \tilde{\chi}_{1}^{ \pm}$, and with the three lepton final states along with missing energy [74-76]. However, we observe that, for the same set of parameters, the rates corresponding to signal final states are higher for top-squark pair production via strong

$$
\begin{array}{cc}
1.5 \leq \tan \beta \leq 60, & 30 \leq \mathrm{M}_{1} \leq 1000, \\
100 \leq|\mu| \leq 1500, & 100 \leq \mathrm{m}_{\mathrm{A}} \leq 1500,
\end{array}
$$

while the other gaugino mass parameter is fixed as

$$
\mathrm{M}_{3}=3 \mathrm{TeV} \text {. }
$$

First two generations squark masses are assumed to be

$$
\mathrm{M}_{\mathrm{Q}_{1,2}}=3 \mathrm{TeV} .
$$

The A term corresponding to the third generation quark $\left(\mathrm{A}_{\mathrm{t}}\right)$ plays an important role in determining the lightest $\mathrm{CP}$ even SM-like Higgs boson mass, and it is varied in the range

$$
-6 \mathrm{TeV} \leq \mathrm{A}_{\mathrm{t}} \leq 6 \mathrm{TeV} .
$$

All the slepton masses of the first two generations are fixed to $2 \mathrm{TeV}$. While performing the scan, each model point is tested with PLANCK [4] data [Eq. (1.1)] and limits from direct searches [11-20]. We focus only on the LSP of the mass range $\sim 50-500 \mathrm{GeV}$. The presence of SM-like Higgs boson (h), with mass $125 \pm 3 \mathrm{GeV}$ is also ensured. Other absolute constraints from LEP [83], for example, $\mathrm{m}_{\tilde{\chi}_{1}^{ \pm}} \geq$ $103.5 \mathrm{GeV}$ and $\mathrm{m}_{\mathrm{H}^{ \pm}}>78.6 \mathrm{GeV}$ are imposed. In addition, HiggsBounds-5.5.0 [84-88] is used to check the Higgs couplings and related measurements. The exclusion of topsquark-neutralino mass plane predicted by CMS [89-92] and ATLAS [93-96] experiments are also examined using the SModelS-1.2.3 package [97,98]. Generally the SMS model with $\operatorname{BR}\left(\tilde{\mathrm{t}}_{1} \rightarrow \tilde{\chi}_{1}^{0}+\mathrm{t}\right)=100 \%$ is used to interpret data. Whereas, in our scenario, $\operatorname{BR}\left(\tilde{t}_{1} \rightarrow \tilde{\chi}_{1}^{0}+\mathrm{t}\right) \sim 10 \%$ implies interaction than the case of electroweak associated production.

\section{A. Numerical scan}

In order to identify the region of parameter space of our interest we perform an illustrative numerical scan of all relevant parameters. This scan is carried out using SUSPECT [77] to calculate the spectrum for a given set of input parameters, and then interfacing with SUSYHIT [78] to obtain respective branching fractions of SUSY particle decays. Also MicrOMEGAs [79-82] is interfaced for the calculation of DM related observables and then checking the constraints.

We have set the ranges of the most relevant parameters, including third generation soft squark masses $\left(\mathrm{M}_{\mathrm{Q}_{3}}, \mathrm{M}_{\mathrm{t}_{\mathrm{R}}}\right.$ ), in the random scan (every unit is in $\mathrm{GeV}$, wherever applicable):

$$
\begin{aligned}
& 100 \leq \mathrm{M}_{2} \leq 3000, \\
& 600 \leq \mathrm{M}_{\mathrm{Q}_{3}} \leq 2500, \quad 600 \leq \mathrm{M}_{\mathrm{t}_{\mathrm{R}}} \leq 2500,
\end{aligned}
$$

much weaker exclusion limits and consequently relatively light top squarks $\left(\mathrm{m}_{\tilde{t}_{1}} \sim 700 \mathrm{GeV}\right)$ are also found to be allowed. Performing the scan, Fig. 1 (left) is plotted, where mainly the relic density and DD constraints are relaxed to show the effect of the compositions of $\tilde{\chi}_{1}^{0}$ on the relic density and DD measurements.

Few representative benchmark points (BP) are chosen (see Table I), which are consistent with all constraints mentioned above. These BPs are used to obtain the signal sensitivities by performing the simulation of our proposed signal process. These BPs primarily represent two scenarios, namely "mild-tempered neutralino" and "blind spots." But under these broad pictures, they also encompass compressed and noncompressed spectrum corresponding to various choices of mass differences, $\Delta \mathrm{m}_{1}=\mathrm{m}_{\tilde{t}_{1}}-\left(\mathrm{m}_{\mathrm{t}}+\mathrm{m}_{\tilde{\chi}_{23}^{0}}\right)$ and $\Delta \mathrm{m}_{2}=\mathrm{m}_{\tilde{\chi}_{2,3}^{0}}-\mathrm{m}_{\tilde{\chi}_{1}^{0}}$.

Notice also that, for all cases of BPs, $\mathrm{m}_{\tilde{t}_{1}}$ varies from 600 $1700 \mathrm{GeV}$ and for all cases $\operatorname{BR}\left(\tilde{t}_{1} \rightarrow \tilde{\chi}_{1}^{0}+\mathrm{t}\right)$ is subdominant, while $\tilde{\chi}_{2,3}^{0} \rightarrow \tilde{\chi}_{1}^{0}+\mathrm{h}$ is dominant. Performing the simulation of signal and backgrounds, signal sensitivities are presented for all these BPs.

\section{SIGNAL AND BACKGROUND}

As discussed before, we consider the following process where the lightest neutralino originates from the decay of second and third lightest neutralino $\left(\tilde{\chi}_{2,3}^{0}\right)$ produced via topsquark production as shown below.

Since in this scenario, the $\tilde{\chi}_{2}^{0} / \tilde{\chi}_{3}^{0}$ are dominantly Higgsino-like, hence $\operatorname{BR}\left(\tilde{t}_{1} \rightarrow t+\tilde{\chi}_{2,3}^{0}\right)$ is larger than the 
$\mathrm{BR}\left(\tilde{\mathrm{t}}_{1} \rightarrow \mathrm{t}+\tilde{\chi}_{1}^{0}\right)$. Subsequently, the higher neutralino state (either $\tilde{\chi}_{2}^{0}$ or $\tilde{\chi}_{3}^{0}$ ) dominantly decays to SM-like Higgs boson and $\tilde{\chi}_{1}^{0}$. Here $\mathrm{X} \equiv \tilde{\chi}_{1}^{0}, \tilde{\chi}_{2,3}^{0}$ leads to a $\tilde{\chi}_{1}^{0}$ accompanied by either $\mathrm{Z}$ or $\mathrm{h}$ in the final state. We focus only on a single Higgs boson in the final state. However, we found that the contribution of di-Higgs boson events in the signal is negligible. The $b \bar{b}$ channel of Higgs boson decay is considered owing to its higher BR and comparatively easy to reconstruct its mass. The pair of lightest neutralinos escape the detector leading to a huge amount of missing energy in the final state. Moreover, there is another pair of $b$ jets originating from two top quarks. Hence, the final state of the signal event is characterized by

$$
\mathrm{h}_{\mathrm{b} \overline{\mathrm{b}}}+\ell+\mathbb{E}_{\mathrm{T}}+(\geq 1) \mathrm{b}-\text { jets, } \quad \ell=\mathrm{e}, \mu .
$$

We found that the contribution of di-Higgs production to the signal event is negligible. It is known that QCD is the main source of background corresponding to any pure hadronic final state. Hence, in order to eliminate it, the leptonic decay of one of the top quarks is considered. We require the presence of only one lepton in the final state. The other dominant SM backgrounds are

$$
\mathrm{pp} \rightarrow \mathrm{t} \overline{\mathrm{t}}(1 \ell), \quad \overline{\mathrm{t}}(2 \ell), \quad \overline{\mathrm{t}} \mathrm{t}, \quad \quad \overline{\mathrm{t} Z}, \quad \overline{\mathrm{t}} \mathrm{b} \overline{\mathrm{b}},
$$

where the combination of two bs coming from the top, h, Z, or gluon splitting mimics the signal $b$ jets from Higgs decay. The lepton and $\mathscr{E}_{\mathrm{T}}$ arise from the semileptonic decay of one of the top quarks, while the other top decays hadronically.

It is to be noted that, in the signal events, the angular separation between two $b$ jets depends on the boost of Higgs boson, which is determined by the mass differences, $\Delta \mathrm{m}_{1}=$ $\mathrm{m}_{\tilde{\mathrm{t}}_{1}}-\left(\mathrm{m}_{\mathrm{t}}+\mathrm{m}_{\tilde{\chi}_{2,3}^{0}}\right)$ and $\Delta \mathrm{m}_{2}=\mathrm{m}_{\tilde{\chi}_{2,3}^{0}}-\mathrm{m}_{\tilde{\chi}_{1}^{0}}$. Accordingly, we simulate signal events in resolved and nonresolved categories depending on the boost of Higgs boson. In Table I, BP1-BP5 correspond to the nonresolved category while BP6-BP9 represent the resolved one. For the boosted case, two b jets likely to appear as a single fat jet, which we refer to as the "Higgs jet (HJ)" now onwards.

The PYTHIA $8[99,100]$ is used to generate $\overline{\mathrm{t}}(1 \ell), \mathrm{t} \overline{\mathrm{t}}(2 \ell)$ events, while the other background processes are generated using MadGraph5-aMC@NLO-2.7.3 [101] interfacing with PYTHIA 8, for showering and hadronization. Signal events are generated in MadGraph5-aMC@NLO-2.7.3 using UFO for the MSSM (MSSM-SLHA2), where the parameter card is generated from SLHA file [102], obtained from

TABLE I. Masses, branching fractions, DM observables for a few representative BPs and labeled those corresponding to the BS scenario. Energy units are in $\mathrm{GeV}$, wherever applicable.

\begin{tabular}{lccccccccc}
\hline \hline & BP1 & BP2 & BP3 & BP4 $(\mathbf{B S})$ & BP5 $(\mathbf{B S})$ & BP6 & BP7 & BP8 $(\mathbf{B S})$ & BP9 $(\mathbf{B S})$ \\
\hline $\mathrm{M}_{1}$ & 60.8 & 58.5 & 274.2 & 334.1 & 296.4 & 204.9 & 352.7 & 238.4 & 248.4 \\
$\mathrm{M}_{2}$ & 2784.4 & 2102.4 & 2719.2 & 1438.5 & 1494.1 & 1093.6 & 1860.2 & 1561.4 & 1071.0 \\
$\mu$ & 655.6 & 793.6 & 984.1 & -789.8 & -717.5 & -489.1 & -610.2 & -414.2 & -539.9 \\
$\mathrm{~m}_{\mathrm{A}}$ & 1252.7 & 953.2 & 584.1 & 712.7 & 585.6 & 453.9 & 762.1 & 459.3 & 543.8 \\
$\tan \beta$ & 7.5 & 6.0 & 6.6 & 6.1 & 6.2 & 5.0 & 5.8 & 6.3 & 6.7 \\
$\mathrm{M}_{\mathrm{Q}_{3}}$ & 856.2 & 1102.2 & 2277.6 & 1024.8 & 1544.2 & 770.1 & 824.4 & 765.8 & 811.5 \\
$\mathrm{M}_{\mathrm{t}_{\mathrm{R}}}$ & 3552.0 & 1889 & 1688.8 & 2403.3 & 2061.9 & 2381.8 & 2596.2 & 2088.9 & 2634.1 \\
$\mathrm{~m}_{\tilde{\mathrm{t}}_{1}}$ & 954 & 1059 & 1675 & 1038 & 1475 & 688 & 804 & 635 & 765 \\
$\mathrm{~m}_{\tilde{\chi}_{3}^{0}}$ & 666 & 802 & 996 & 800 & 729 & 494 & 620 & 424 & 550 \\
$\mathrm{~m}_{\tilde{\chi}_{2}^{0}}$ & 666 & 800 & 994 & 796 & 725 & 499 & 618 & 422 & 545 \\
$\mathrm{~m}_{\tilde{\chi}_{1}^{0}}$ & 59 & 58 & 272 & 335 & 295 & 207 & 354 & 238 & 249 \\
$\mathrm{~m}_{\tilde{\chi}_{1}^{ \pm}}$ & 664 & 799 & 993 & 795 & 725 & 495 & 618 & 420 & 545 \\
$\mathrm{~m}_{\mathrm{h}}$ & 125 & 123 & 123 & 125 & 124 & 123 & 123 & 124 & 125 \\
$\mathrm{~m}_{\mathrm{H}}$ & 1253 & 953 & 584 & 713 & 584 & 454 & 763 & 460 & 544 \\
$\mathrm{~N}_{11}^{2}$ & 0.995 & 0.996 & 0.996 & 0.996 & 0.996 & 0.99 & 0.99 & 0.976 & 0.99 \\
$\mathrm{~N}_{13}^{2}+\mathrm{N}_{14}^{2}$ & 0.005 & 0.003 & 0.003 & 0.004 & 0.004 & 0.01 & 0.01 & 0.023 & 0.01 \\
$\Omega \mathrm{h}^{2}$ & 0.129 & 0.122 & 0.119 & 0.112 & 0.121 & 0.110 & 0.117 & 0.119 & 0.110 \\
$\sigma_{\mathrm{SI}}\left(10^{-11} \mathrm{pb}\right)$ & 5.1 & 5.2 & 10 & 0.009 & 0.02 & 1.9 & 2.4 & 0.69 & 0.002 \\
$\sigma_{\mathrm{SD}}(\mathrm{p})\left(10^{-7} \mathrm{pb}\right)$ & 7.2 & 3.2 & 1.6 & 5.2 & 7.5 & 32 & 21 & 100 & 26 \\
$\sigma_{\mathrm{SD}}(\mathrm{n})\left(10^{-7} \mathrm{pb}\right)$ & 5.7 & 2.5 & 1.3 & 4.1 & 5.8 & 25 & 12 & 78 & 20 \\
$\mathrm{BR}\left(\tilde{\mathfrak{t}}_{1} \rightarrow \tilde{\chi}_{1}^{0}+\mathrm{t}\right)$ & 0.05 & 0.11 & 0.16 & 0.08 & 0.05 & 0.11 & 0.15 & 0.08 & 0.08 \\
$\mathrm{BR}\left(\tilde{\mathrm{t}}_{1} \rightarrow \tilde{\chi}_{2}^{0}+\mathrm{t}\right)$ & 0.49 & 0.31 & 0.20 & 0.37 & 0.34 & 0.32 & 0.33 & 0.34 & 0.33 \\
$\mathrm{BR}\left(\tilde{\mathrm{t}}_{1} \rightarrow \tilde{\chi}_{3}^{0}+\mathrm{t}\right)$ & 0.42 & 0.51 & 0.22 & 0.49 & 0.43 & 0.49 & 0.45 & 0.52 & 0.50 \\
$\mathrm{BR}\left(\tilde{\chi}_{2}^{0} \rightarrow \tilde{\chi}_{1}^{0}+\mathrm{h}\right)$ & 0.70 & 0.73 & 0.70 & 0.73 & 0.72 & 0.85 & 0.83 & 0.78 & 0.75 \\
$\mathrm{BR}\left(\tilde{\chi}_{3}^{0} \rightarrow \tilde{\chi}_{1}^{0}+\mathrm{h}\right)$ & 0.28 & 0.27 & 0.12 & 0.24 & 0.25 & 0.04 & 0.12 & 0.09 & 0.19 \\
\hline \hline
\end{tabular}


SUSYHIT, corresponding to each BP. The same SLHA file is used for subsequent showering of signal events in PYTHIA 8. Detector effects are taken into account by passing all signal and background events through DELPHES-3.4.2 [103] using the CMS detector card. ${ }^{1}$

In the simulation, the following selections are imposed, where objects are selected using DELPHES inputs.

(1) Lepton selection: leptons are selected with $\mathrm{p}_{\mathrm{T}}>$ $20 \mathrm{GeV}$ and $|\eta|<2.5$. Isolation is ensured using mini-isolation criteria by checking e-flow objects of DELPHES as follows [104]:

$$
\frac{\sum \mathrm{p}_{\mathrm{T}}^{\mathrm{R}<\mathrm{r}}}{\mathrm{p}_{\mathrm{T}, \ell}}<\mathrm{I}, \quad \ell=\mathrm{e}, \mu .
$$

Here $\mathrm{r}=\frac{10.0}{\mathrm{p}_{\mathrm{T}, \ell}}$ and $\mathrm{I}=0.12$ and 0.25 for e and $\mu$, respectively.

(2) Missing transverse momentum $\left(\mathscr{E}_{\mathrm{T}}\right)$ : the missing transverse momentum is constructed by taking the resultant momenta of all visible particles and then reversing the direction, i.e., $\overrightarrow{\mathrm{p}}_{\mathrm{T}}=-\sum \overrightarrow{\mathrm{p}}_{\mathrm{T}}^{\mathrm{i}}$, where $i$ runs over all constructed visible collection from the detector. A cut $\mathbb{E}_{\mathrm{T}}>200(150) \mathrm{GeV}$ is imposed for events in the nonresolved (resolved) category.

(3) HJ selection: the reconstruction of $\mathrm{HJ}$ is performed in two ways depending on the boost of the Higgs boson, i.e., resolved and nonresolved categories, as described below.

(i) $\mathrm{HJ}$ in a nonresolved category: at first, fat jets are constructed taking inputs from DELPHES, using FASTJET3.3.2 [105] with Cambridge-Aachen [106] algorithm and $R=1.0$. Minimum $\mathrm{p}_{\mathrm{T}}$ of the fat jets is set to be $100 \mathrm{GeV}$. These fat jets are then passed through mass-drop tagger $[107,108]$ with $\mu=0.667$ and $\mathrm{y}_{\text {cut }}>0.09$ to remove contamination due to soft radiation. The subjets of the "tagged fat jet" are further matched with the b quarks of the event, which are selected within $|\eta|<2.5$ and with a matching cone $\Delta \mathrm{R}<0.3$. When both the subjets are found to be b-like, we call the tagged fat jet the $\mathrm{HJ}\left(\mathrm{J}_{\mathrm{bb}}\right)$. We also checked the presence of B hadron in the b-like subjets and found that, for about $95 \%$ cases, it exists.

(ii) Resolved category: in this case, jets, subject to cuts $\mathrm{p}_{\mathrm{T}}^{\mathrm{j}}>20 \mathrm{GeV}$ and $|\eta|<4.0$, are constructed from e-flow objects of DELPHES, using FASTJET3.3.2 [105], but with an anti- $\mathrm{k}_{\mathrm{T}}$ [109] algorithm and with a jet size parameter $R=0.5$. Using the same technique as above, by matching jets with $b$ quarks of the event, b-like jets are identified. The pair of b-like jets that construct

\footnotetext{
${ }^{1}$ Results are checked with the ATLAS card as well, and no appreciable change is observed.
}

the invariant mass closest to Higgs boson mass within the range $100 \mathrm{GeV} \leq \mathrm{m}_{\mathrm{HJ}} \leq 150 \mathrm{GeV}$ is identified as $\mathrm{HJ}$, and the resultant four momentum of the two jets is regarded as the momentum of HJ.

(4) Other jets and $b$ jets: this selection also differs according to two categories.

(i) Jets in a nonresolved category: once $\mathrm{HJ}$ is constructed, the remaining hadrons are used to construct regular QCD jets through FASTJET3.3.2 with an anti- $\mathrm{k}_{\mathrm{T}}$ algorithm setting $\mathrm{R}=0.5$. Out of these jets, b-like jets are identified by matching the technique with the remaining set of $b$ quarks in the event, which are not part of $\mathrm{J}_{\mathrm{bb}}$.

(ii) Jets in resolved category: the two b jets, which are found to be related to the $\mathrm{HJ}$, are removed from the list of jets and $\mathrm{b}$ jets, and this new list is used further.

Furthermore, to suppress backgrounds, we impose few more selection cuts. For example, the transverse mass between lepton and $\boldsymbol{E}_{\mathrm{T}}$, defined as

$\mathrm{m}_{\mathrm{T}}\left(\ell, \mathbb{E}_{\mathrm{T}}\right)=\sqrt{2 \times \mathrm{p}_{\mathrm{T}}^{\ell} \times \mathbb{E}_{\mathrm{T}} \times\left(1-\cos \phi\left(\ell, \mathbb{E}_{\mathrm{T}}\right)\right)}$,

is restricted by $\mathrm{M}_{\mathrm{W}}$ for all semileptonic $\mathrm{t} \overline{\mathrm{t}}$ background events as seen in the $\mathrm{m}_{\mathrm{T}}$ distribution presented in Fig. 3 (left) along with signal events corresponding to two BPs. On the contrary, for signal events, having a large $\mathbb{E}_{\mathrm{T}}$ due to neutralinos, which is also not correlated with the lepton coming from $t \bar{t}$ decay, is expected to have a more wide $\mathrm{m}_{\mathrm{T}}$ distribution without any peaks [see Fig. 3 (left)]. Hence a cut $\mathrm{m}_{\mathrm{T}}\left(\ell, E_{\mathrm{T}}\right) \geq 110 \mathrm{GeV}$ turns out to be very effective in eliminating a certain fraction of the background.

Another discriminating variable is $\mathrm{H}_{\mathrm{T}}$, defined as the scalar sum of $\mathrm{p}_{\mathrm{T}}$ of all jets except those that constitute HJ. For signal events, a larger number of harder jets exist leading to higher $\mathrm{H}_{\mathrm{T}}$ as seen from the distribution shown in Fig. 3 (right). A cut $\mathrm{H}_{\mathrm{T}} \geq 500 \mathrm{GeV}$ turns out to be useful to reject background events substantially.

In the case of the nonresolved category, the mass distribution of $\mathrm{J}_{\mathrm{bb}}$ shows a clear peak at $\sim 125 \mathrm{GeV}$, which is absent in most of the backgrounds, and very small for tth as shown in Fig. 4. Thus the selection of $\mathrm{m}_{\mathrm{Jb}}>100 \mathrm{GeV}$ is found to be useful in eliminating significant background events. In the resolved category case, this mass requirement is already imposed while constructing HJ. The presence of HJ with a specific mass requirement is a very important feature of our signal and helps to eliminate almost all the $t \bar{t}$ backgrounds by enormous amount except tth process where the source of $\mathrm{J}_{\mathrm{bb}}$ is same as the signal.

Signal events are simulated for 9 BPs which are chosen in such a way that BP1-BP5 represent the nonresolved cases, whereas BP6-BP9 correspond to the resolved category. The BPs labeled as "BS" in the parenthesis 

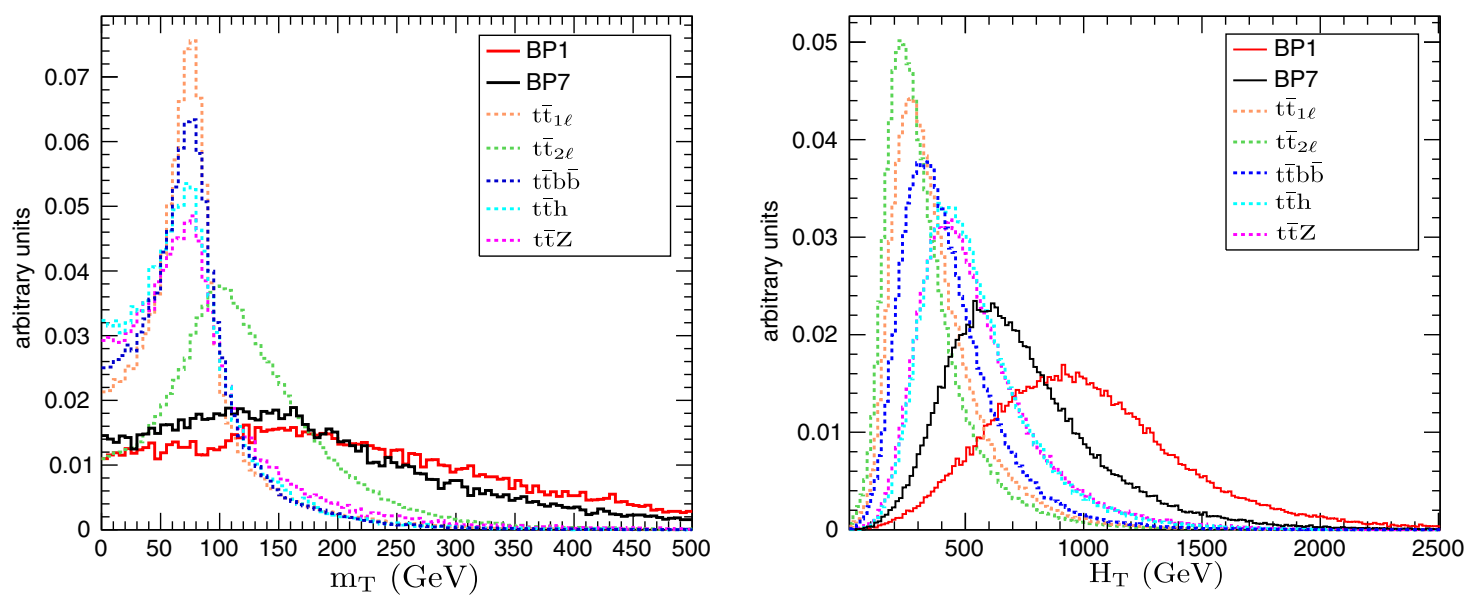

FIG. 3. Transverse mass between [Eq. (3.4)] a lepton and $\mathbb{E}_{\mathrm{T}}$ (left) and $\mathrm{H}_{\mathrm{T}}$ (right) for BP1, BP7, and dominating backgrounds.

correspond to a BS scenario. In Table II, the cross section yields the signal and background processes for the nonresolved categories and are presented after imposing selection cuts. The first row presents the leading order (LO) cross sections of each processes, computed by MadGraph5aMC@NLO-2.7.3, at the center of mass energy $\sqrt{\mathrm{s}}=13 \mathrm{TeV}$, using NNPDF23LO [110] for parton distribution and choosing the dynamic QCD scale $\left(Q^{2}=\sqrt{\left[m_{\tilde{t}_{1}}^{2}+p_{T}^{2}\left(\tilde{t}_{1}\right)\right]\left[m_{\tilde{t}_{1}}^{2}+p_{T}^{2}\left(\tilde{t}_{1}\right)\right]}\right)$. Higher order effects are taken into account by multiplying respective $\mathrm{K}$ factors $\left(\mathrm{K}=\frac{\sigma_{\mathrm{NLO}}}{\sigma_{\mathrm{LO}}}\right)$. A $\mathrm{K}$ factor of 1.4 is used for top-squark pair production (for NNPDF31LO) [111] and $\overline{\mathrm{t}}$ [112,113].

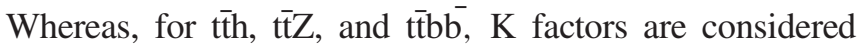
to be 1.2 [114], 1.35 [115], and 1.8 [116], respectively. As indicated in the table, the $\mathrm{m}_{\mathrm{J}_{\mathrm{bb}}}$ cut is very useful to eliminate backgrounds significantly. In addition, the $\mathrm{m}_{\mathrm{T}}$ cut also kills backgrounds substantially.

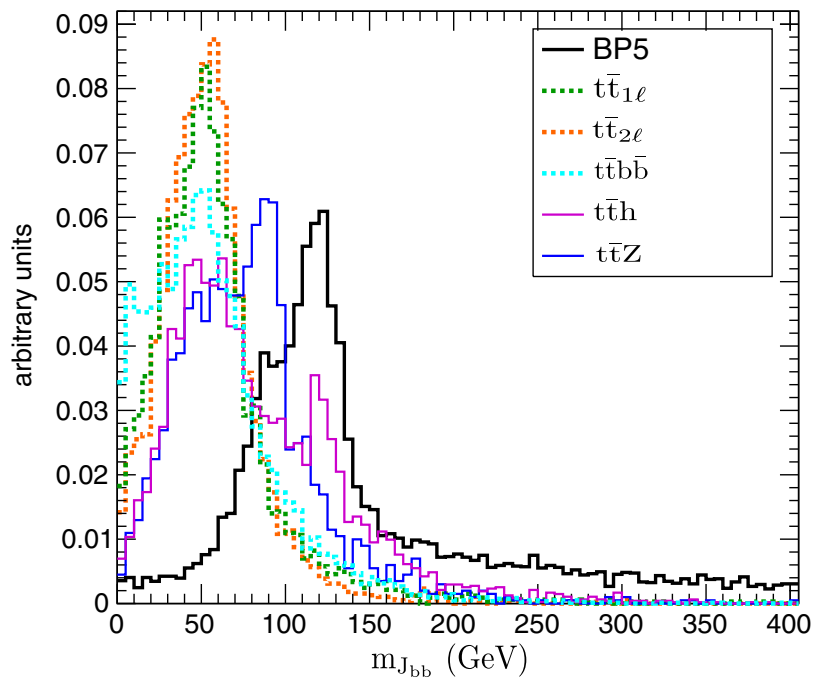

FIG. 4. Reconstructed mass of $\mathrm{HJ}$ for a representative signal (BP5) point and dominant backgrounds.
Similarly, cross section yields for the resolved category are presented in Table III. It is clear that the selection of HJ, in this case, is not as efficient as the nonresolved category, but it still has good discriminating power. In general, overall signal acceptance efficiency is $1-2 \%$; while, for overall backgrounds, it is found to be $0.0001 \%$ for the nonresolved category and $0.007 \%$ for the resolved category. The total cross sections of background events are found to be $0.232 \mathrm{fb}$ for the nonresolved category and $16.2 \mathrm{fb}$ for the resolved cases, respectively. Finally, the signal sensitivities $\left(\frac{\mathrm{S}}{\sqrt{\mathrm{S}+\mathrm{B}}}\right)$ are presented in Table IV for two high luminosity options $\mathcal{L}=300 \mathrm{fb}^{-1}$ and $3000 \mathrm{fb}^{-1}$. It is to be noted that, for the nonresolved category, the sensitivities are $\sim 2-3 \sigma$ for $\mathcal{L}=300 \mathrm{fb}^{-1}$, whereas they are large $(\sim 5-8 \sigma)$ for the resolved category, mainly because of the high production cross sections, due to smaller top-squark masses. The tiny sensitivities for BP3 and BP5 can be attributed to a very low top-squark production cross section because of its higher masses. Assuming 10\% background uncertainty the sensitivity for the BPs in resolved category drops by $\sim 7 \%$ and for the nonresolved category, it reduces by about $0.1 \%$.

Though we obtain reasonable signal sensitivity in the resolved category, the acceptance efficiencies for backgrounds, in that case, are not appreciably small as in the nonresolved category in the cut-based method. In order to improve further, we carry out multivariate analysis (MVA) based on a boosted decision tree method within the framework of TMVA $[117,118]$.

\section{A. Multivariate analysis}

The basic idea of MVA [117-121] is to examine patterns in multidimensional data by considering several variables at once. Several kinematical variables are constructed, keeping in mind the features of signal events for training purposes. Depending upon the performances of those variables, we use 13 of those for the nonresolved category and 15 for the resolved category to train signal and 
TABLE II. Cross section (in fb) yields after each set of selection cuts for signal points in the nonresolved category and background processes.

\begin{tabular}{|c|c|c|c|c|c|c|c|c|c|c|}
\hline & $\mathrm{BP} 1$ & $\mathrm{BP} 2$ & BP3 & $\mathrm{BP} 4(\mathbf{B S})$ & $\mathrm{BP} 5(\mathbf{B S})$ & $\overline{\mathrm{t}}(1 \ell)$ & $\overline{\mathrm{tt}}(2 \ell)$ & tth & $\mathrm{t} \overline{\mathrm{t} Z}$ & $\bar{t} \bar{b} \bar{b}$ \\
\hline Cross section (LO) (fb) & 6 & 3 & 0.06 & 3 & 0.18 & 178500 & 36000 & 400 & 584 & 13700 \\
\hline $\mathbb{E}_{\mathrm{T}}>200 \mathrm{GeV}$ & 4.9 & 2.5 & 0.054 & 2.4 & 0.17 & 2695 & 592.5 & 12.6 & 38.8 & 186.7 \\
\hline No. of $\ell=1$ & 1.5 & 0.73 & 0.02 & 0.7 & 0.05 & 1419 & 291.2 & 5.1 & 11.5 & 71.1 \\
\hline No. of $\mathrm{J}_{\mathrm{bb}}=1$ & 0.4 & 0.2 & 0.004 & 0.2 & 0.014 & 33.8 & 12.1 & 1.1 & 0.8 & 10.6 \\
\hline $\mathrm{m}_{\mathrm{J}_{\mathrm{bb}}}>100 \mathrm{GeV}$ & 0.3 & 0.15 & 0.003 & 0.14 & 0.01 & 11.6 & 2.7 & 0.7 & 0.3 & 2.6 \\
\hline No. of $b$ jets $\geq 1$ & 0.15 & 0.07 & 0.001 & 0.07 & 0.0036 & 1.0 & 0.25 & 0.3 & 0.1 & 0.8 \\
\hline $\mathrm{H}_{\mathrm{T}}>500 \mathrm{GeV}$ & 0.1 & 0.05 & 0.0008 & 0.05 & 0.003 & 0.25 & 0.07 & 0.1 & 0.05 & 0.1 \\
\hline $\mathrm{m}_{\mathrm{T}}\left(\ell, \boldsymbol{E}_{\mathrm{T}}\right) \geq 110 \mathrm{GeV}$ & 0.08 & 0.043 & 0.0007 & 0.04 & 0.003 & 0.04 & 0.07 & 0.02 & 0.006 & 0.02 \\
\hline$\sigma \times \mathrm{K}$ factor & 0.12 & 0.06 & 0.001 & 0.056 & 0.004 & 0.06 & 0.1 & 0.024 & 0.008 & 0.04 \\
\hline
\end{tabular}

TABLE III. Same as in Table II, but for resolved category.

\begin{tabular}{|c|c|c|c|c|c|c|c|c|c|}
\hline & BP6 & BP7 & $\mathrm{BP} 8(\mathbf{B S})$ & $\mathrm{BP} 9(\mathbf{B S})$ & $\mathrm{t} \mathrm{t}(1 \ell)$ & $\mathrm{t} \mathrm{t}(2 \ell)$ & $\mathrm{t} \overline{\mathrm{th}}$ & $\mathrm{t} \overline{\mathrm{t} Z}$ & $\overline{\mathrm{t}} \mathrm{b} \overline{\mathrm{b}}$ \\
\hline Cross section (fb) & 53 & 19 & 88 & 27 & 178500 & 36000 & 400 & 584 & 13700 \\
\hline $\mathbb{E}_{\mathrm{T}}>150 \mathrm{GeV}$ & 36.4 & 14.1 & 51.2 & 20.5 & 8560 & 2100 & 31.3 & 76.5 & 555.6 \\
\hline No. of $\ell=1$ & 9.8 & 3.8 & 13.9 & 5.6 & 4364 & 1050 & 12.2 & 23.8 & 204.1 \\
\hline No. of $\mathrm{J}_{\mathrm{bb}}=1$ & 3.9 & 1.5 & 5.4 & 2.3 & 564.2 & 145.9 & 4.6 & 3.9 & 53.3 \\
\hline No. of $b$ jets $\geq 1$ & 2.9 & 1.1 & 4.0 & 1.8 & 49.5 & 11.0 & 3.6 & 1.3 & 35.7 \\
\hline $\mathrm{H}_{\mathrm{T}}>500 \mathrm{GeV}$ & 1.7 & 0.7 & 2.3 & 1.2 & 22.3 & 3.6 & 1.7 & 0.6 & 11.8 \\
\hline $\mathrm{m}_{\mathrm{T}}\left(\ell, \mathscr{E}_{\mathrm{T}}\right) \geq 110 \mathrm{GeV}$ & 1.2 & 0.5 & 1.5 & 0.9 & 5.3 & 2.4 & 0.4 & 0.15 & 2.6 \\
\hline$\sigma \times \mathrm{K}$ factor & 1.7 & 0.7 & 2.1 & 1.22 & 7.5 & 3.36 & 0.43 & 0.20 & 4.7 \\
\hline
\end{tabular}

TABLE IV. Signal significances $\left(\frac{\mathrm{S}}{\sqrt{\mathrm{S}+\mathrm{B}}}\right)$ for two luminosity options.

\begin{tabular}{|c|c|c|c|c|c|c|c|c|c|}
\hline \multirow[b]{2}{*}{ Luminosity $\left(\mathrm{fb}^{-1}\right)$} & \multicolumn{5}{|c|}{ Nonresolved category } & \multicolumn{4}{|c|}{ Resolved category } \\
\hline & BP1 & BP2 & BP3 & BP4 & BP5 & BP6 & BP7 & BP8 & BP9 \\
\hline 300 & 3.5 & 2.0 & 0.035 & 1.8 & 0.14 & 7 & 2.9 & 8.5 & 5.0 \\
\hline 3000 & 11 & 6 & 0.1 & 6 & 0.44 & 22 & 9 & 27 & 16 \\
\hline
\end{tabular}

TABLE V. Rank of variables in MVA for nonresolved category corresponding to BP5.

\begin{tabular}{|c|c|c|}
\hline Rank & Variable & Description \\
\hline 1 & $\mathrm{~m}_{\mathrm{h}}$ & Mass of $\mathrm{J}_{\mathrm{bb}}$ \\
\hline 2 & HT & Scalar sum of $\mathrm{p}_{\mathrm{T}}$ of all jets outside $\mathrm{J}_{\mathrm{bb}}$ \\
\hline 3 & $E_{\mathrm{T}}$ & Missing $\mathrm{p}_{\mathrm{T}}$ \\
\hline 4 & $\Delta \mathrm{R}\left(\mathscr{E}_{\mathrm{T}}, \mathrm{J}_{\mathrm{bb}}\right)$ & $\Delta \mathrm{R}$ between $\mathbb{E}_{\mathrm{T}}$ and $\mathrm{J}_{\mathrm{bb}}$ \\
\hline 5 & $\mathrm{p}_{\mathrm{T}}\left(\mathrm{J}_{\mathrm{bb}}\right)$ & $\mathrm{p}_{\mathrm{T}}$ of $\mathrm{J}_{\mathrm{bb}}$ \\
\hline 6 & $\mathrm{p}_{\mathrm{T}}(\ell)$ & $\mathrm{p}_{\mathrm{T}}$ of leading lepton \\
\hline 7 & $\Delta \mathrm{R}\left(\mathrm{b}_{1}, \mathrm{~J}_{\mathrm{bb}}\right)$ & $\begin{array}{l}\Delta \mathrm{R} \text { between leading } \mathrm{b} \text { jet (outside } \mathrm{J}_{\mathrm{bb}} \text { ) } \\
\text { and } \mathrm{J}_{\mathrm{bb}}\end{array}$ \\
\hline 8 & $\Delta \mathrm{R}\left(\mathscr{E}_{\mathrm{T}}, \mathrm{j}\right)$ & $\Delta \mathrm{R}$ between $\mathscr{E}_{\mathrm{T}}$ and leading jet outside $\mathrm{J}_{\mathrm{bb}}$ \\
\hline 9 & $\mathrm{~N}$ jets & Number of jets outside $\mathrm{J}_{\mathrm{bb}}$. \\
\hline 10 & $\mathrm{M}_{\mathrm{T}}\left(\ell, \boldsymbol{E}_{\mathrm{T}}\right)$ & $\begin{array}{l}\text { Transverse mass of leading } \mathrm{p}_{\mathrm{T}} \\
\text { lepton and } \mathscr{E}_{\mathrm{T}}\end{array}$ \\
\hline 11 & $\mathrm{~N}(\ell)$ & Number of leptons \\
\hline 12 & $\mathrm{p}_{\mathrm{T}}(\mathrm{b}$ jet $)$ & $\mathrm{p}_{\mathrm{T}}$ of leading $\mathrm{b}$ jet outside $\mathrm{J}_{\mathrm{bb}}$ \\
\hline 13 & $\mathrm{~N}(\mathrm{~b}$ jet $)$ & Number of $b$ jets, outside $J_{b b}$ \\
\hline
\end{tabular}

TABLE VI. Rank of variables in MVA for resolved categories corresponding to BP7.

\begin{tabular}{|c|c|c|}
\hline Rank & Variable & Description \\
\hline 1 & $E_{\mathrm{T}}$ & Missing $\mathrm{p}_{\mathrm{T}}$ \\
\hline 2 & $\mathrm{~m}_{\mathrm{h}}$ & Mass of $\mathrm{J}_{\mathrm{bb}}$ \\
\hline 3 & $\Delta \mathrm{R}\left(\mathrm{b}_{1}, \mathrm{~b}_{2}\right)$ & $\Delta \mathrm{R}$ between two b jets inside Higgs jet. \\
\hline 4 & $\Delta \mathrm{R}\left(\boldsymbol{E}_{\mathrm{T}}, \mathrm{J}_{\mathrm{bb}}\right)$ & $\Delta \mathrm{R}$ between $\mathscr{E}_{\mathrm{T}}$ and $\mathrm{J}_{\mathrm{bb}}$ \\
\hline 5 & $\mathrm{p}_{\mathrm{T}}\left(\mathrm{b}_{1}\right) / \mathrm{p}_{\mathrm{T}}\left(\mathrm{b}_{2}\right)$ & pT ratio of two $b$ jets inside Higgs jet. \\
\hline 6 & $\Delta \mathrm{R}\left(\boldsymbol{E}_{\mathrm{T}}, \mathrm{j}\right)$ & $\Delta \mathrm{R}$ between $\mathscr{E}_{\mathrm{T}}$ and leading jet \\
\hline 7 & $\Delta \mathrm{R}\left(\mathrm{b}_{1}, \mathrm{~J}_{\mathrm{bb}}\right)$ & $\begin{array}{l}\Delta \mathrm{R} \text { between leading } \mathrm{b} \text { jet } \\
\left(\text { outside } \mathrm{J}_{\mathrm{bb}}\right) \text { and } \mathrm{J}_{\mathrm{bb}}\end{array}$ \\
\hline 8 & $\mathrm{~N}$ jets & Number of outside $\mathrm{J}_{\mathrm{bb}}$. \\
\hline 9 & $\mathrm{HT}$ & Scalar sum of $\mathrm{p}_{\mathrm{T}}$ of all jets outside $\mathrm{J}_{\mathrm{bb}}$ \\
\hline 10 & $\mathrm{p}_{\mathrm{T}}(\ell)$ & $\mathrm{p}_{\mathrm{T}}$ of leading lepton \\
\hline 11 & $\mathrm{M}_{\mathrm{T}}\left(\ell, \mathbb{E}_{\mathrm{T}}\right)$ & $\begin{array}{l}\text { Transverse Mass of leading pT } \\
\text { lepton and } \mathscr{E}_{\mathrm{T}}\end{array}$ \\
\hline 12 & $\mathrm{p}_{\mathrm{T}}\left(\mathrm{J}_{\mathrm{bb}}\right)$ & $\mathrm{p}_{\mathrm{T}}$ of $\mathrm{J}_{\mathrm{bb}}$ \\
\hline 13 & $\mathrm{~N}(\mathrm{~b}$ jet $)$ & Number of $b$ jets, outside $J_{b b}$ \\
\hline 14 & $\mathrm{~N}(\ell)$ & Number of leptons \\
\hline 15 & $\mathrm{p}_{\mathrm{T}}(\mathrm{b}$ jet $)$ & $\mathrm{p}_{\mathrm{T}}$ of leading $\mathrm{b}$ jet outside $\mathrm{J}_{\mathrm{bb}}$ \\
\hline
\end{tabular}



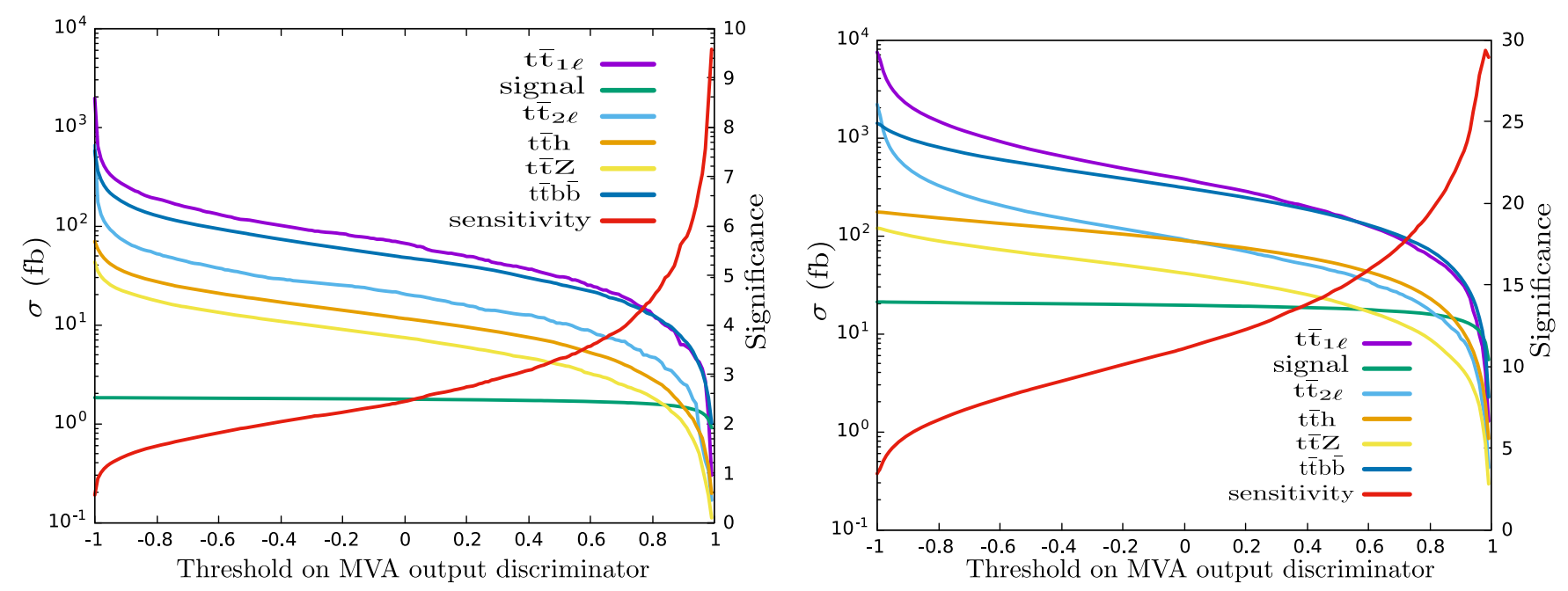

FIG. 5. Signal and background yields as a function of threshold on MVA output discriminator along with the significance of signal corresponding to $\mathcal{L}=300 \mathrm{fb}^{-1}$ for signal point BP1 (left) and BP6 (right).

background samples. The description of those variables are presented in Tables V and VI corresponding to BP5 for nonresolved category and BP7 for the resolved category, respectively.

The first column of these tables shows the ranking of these variables, which represents the relative importance in discriminating signal and backgrounds. The set of variables are the same for all BPs for a given category, but, depending on the kinematics, the ranking of those variables is found to be little different. While doing MVA for each BP, overtraining tests are performed to ensure that there are no significant deviations between the performance of training and testing data.

In Fig. 5, the variation of cross section yields for signal and backgrounds and the signal significance $\left(\frac{\mathrm{S}}{\sqrt{\mathrm{S}+\mathrm{B}}}\right)$ as a function of threshold on MVA output discriminator for luminosity $\mathcal{L}=300 \mathrm{fb}^{-1}$ is presented corresponding to the BP1 for the nonresolved category and BP6 for the resolved category case. It indicates that a sensitivity above $\sim 5 \sigma$ can be achieved for luminosity $\mathcal{L}=300 \mathrm{fb}^{-1}$ corresponding to a cut of the classifier $>0.9$.

Evidently, the achievable signal significance for all the BPs are presented in Table VII for two luminosity options. Clearly, the signal sensitivities are found to be well above $5 \sigma$ at $\mathcal{L}=300 \mathrm{fb}^{-1}$, except for BP3 and BP5, where the production cross section is too low due to a heavier topsquark mass.

\section{SUMMARY}

In the MSSM framework, the lightest neutralino, an LSP of the mass $\sim \mathcal{O}(100) \mathrm{GeV}$, is found to be one of the best suitable DM candidates. However, the constraints from direct DM detection experiments and measurement of the relic density restrict the composition of the physical neutralino states. It is observed that, instead of a pure state, neutralino DM in MSSM is "mild-tempered" where it is bino dominated with a presence of little Higgsino, providing the best DM solution at this mass range. In this scenario, the DM annihilation process takes place via Higgs and gauge bosons where Higgsino content along with dominant bino helps to provide the right relic density. It is to be noted that, eventually the Higgsino composition in the LSP is strongly restricted by the limits of SI DM-nucleon scattering cross section measurements in the direct DM detection experiments, primarily by XENON1T. Considering this DM solution, a numerical scan is performed to identify the range of sensitive parameters, in particular, $\mu$ and $\mathrm{M}_{1}$ in the limit of a very large $\mathrm{M}_{2}$ value. It is found that, with $|\mu|-\mathrm{M}_{1}>\mathrm{M}_{\mathrm{Z}}$, the most preferred ranges are $\mathrm{M}_{1} \sim 50-600 \mathrm{GeV}$ and $\mu \sim 400-1000 \mathrm{GeV}$. Moreover, there is a region of parameter space that is blind to the SI scattering cross section due to the interplay of parameters and cancellation among various amplitudes mediated by the lighter and heavier Higgs bosons. Consequently, in such cases, the Higgsino content in the

TABLE VII. Signal significances $\left(\frac{\mathrm{S}}{\sqrt{\mathrm{S}+\mathrm{B}}}\right)$ for two luminosity options applying MVA.

\begin{tabular}{|c|c|c|c|c|c|c|c|c|c|}
\hline \multirow[b]{2}{*}{ Luminosity $\left(\mathrm{fb}^{-1}\right)$} & \multicolumn{5}{|c|}{ Nonresolved category } & \multicolumn{4}{|c|}{ Resolved category } \\
\hline & BP1 & BP2 & BP3 & BP4 & BP5 & BP6 & BP7 & BP8 & BP9 \\
\hline 300 & 6 & 4.5 & 0.14 & 3.6 & 0.35 & 24 & 9.5 & 27 & 15 \\
\hline 3000 & 19 & 14 & 0.5 & 11 & 1.1 & 75 & 30 & 85 & 47 \\
\hline
\end{tabular}


lightest neutralino is not severely constrained. In mildtempered DM scenario, $\tilde{\chi}_{1}^{0}$ is accompanied with Higgsinolike $\tilde{\chi}_{2,3}^{0}$ and $\tilde{\chi}_{1}^{ \pm}$having masses around $\mu$. It is indeed the case even for the region of parameters corresponding to the BS scenario. Due to the gaugino-Higgsino-Higgs type of coupling, $\tilde{\chi}_{2,3}^{0} \rightarrow \mathrm{h}+\tilde{\chi}_{1}^{0}$ decay rate gets enhanced, leading to an interesting phenomenology at the LHC corresponding to our considered scenario.

We focus on the top-squark pair production to explore the mild-tempered neutralino scenario at the LHC. As $\mathrm{BR}\left(\tilde{\mathrm{t}}_{1} \rightarrow \tilde{\chi}_{1}^{0}+\mathrm{t}\right)$ is very small, $\tilde{\chi}_{1}^{0}$ is indirectly produced through the production of $\tilde{\chi}_{2,3}^{0}$. The presence of SM-Higgs boson in the final state adds an extra advantage to probe this channel. Interestingly, this channel also provides an opportunity to probe the BS scenario. The signal is characterized by one $\mathrm{HJ}$ consisting of b-like jets or subjets, large $\mathbb{E}_{\mathrm{T}}$, one lepton, plus at least one extra b-like jet. The HJ tagging turns out to be very efficient to separate out the signal from the debris of backgrounds. The presence of HJ adds robustness to this signal.

Signal significances are presented for few illustrative BPs including a BS scenario. We observe that for top squarks of the mass range $600-1700 \mathrm{GeV}$, for most of the $\mathrm{BPs}$, a reasonable signal sensitivity $(\sim 3-5 \sigma)$ can be achieved corresponding to $\mathcal{L}=300 \mathrm{fb}^{-1}$ luminosity option, which goes up roughly by a factor of three for
$\mathcal{L}=3000 \mathrm{fb}^{-1}$. Furthermore, we demonstrate that the sensitivities can be increased by employing MVA technique. Remarkably, we notice that, for the above luminosity options and in particular for the resolved category case, the improvement is significant: by a factor of $\sim 3-4$. The signal is detectable even for the $\mathcal{L}=300 \mathrm{fb}^{-1}$ option except for BP3 and BP5 for which top-squark masses are $\sim 1.5 \mathrm{TeV}$. For the center of mass energy $\sqrt{\mathrm{s}}=14 \mathrm{TeV}$, which is the energy option for RUN3 experiment at the LHC, our projected sensitivities are expected to increase by $15-20 \%$ depending on the top-squark masses. A $10 \%$ uncertainty in background estimation reduces sensitivity by about $7 \%$ and $0.1 \%$ for resolved and nonresolved category, respectively. Our analysis shows that both a "mild-tempered" neutralino providing a DM candidate in the framework of MSSM and the BS scenario where the direct search is not sensitive can be detected at the LHC with a reasonable sensitivity for projected luminosity options.

\section{ACKNOWLEDGEMENTS}

The authors are thankful to Shivani Lomte, at affiliation of IISER, Pune (now at University of Wisconsin) for collaborating on this project at an earlier stage. One of the authors, A. R., is thankful to Soham Bhattacharya for useful discussions and suggestions.
[1] S. Rappoccio, The experimental status of direct searches for exotic physics beyond the standard model at the Large Hadron Collider, Rev. Phys. 4, 100027 (2019).

[2] G. Bertone, D. Hooper, and J. Silk, Particle dark matter: Evidence, candidates and constraints, Phys. Rep. 405, 279 (2005).

[3] J. L. Feng, Dark matter candidates from particle physics and methods of detection, Annu. Rev. Astron. Astrophys. 48, 495 (2010).

[4] N. Aghanim et al. (Planck Collaboration), Planck 2018 results. VI. Cosmological parameters, Astron. Astrophys. 641, A6 (2020).

[5] M. Schumann, Direct detection of WIMP dark matter: Concepts and status, J. Phys. G 46, 103003 (2019).

[6] J. M. Gaskins, A review of indirect searches for particle dark matter, Contemp. Phys. 57, 496 (2016).

[7] T. R. Slatyer, Indirect detection of dark matter, in Theoretical Advanced Study Institute in Elementary Particle Physics: Anticipating the Next Discoveries in Particle Physics (2018), pp. 297-353.

[8] A. Boveia and C. Doglioni, Dark matter searches at colliders, Annu. Rev. Nucl. Part. Sci. 68, 429 (2018).

[9] N. Trevisani, Collider searches for dark matter (atlas + cms), Universe 4, 131 (2018).
[10] M. Aaboud et al. (ATLAS Collaboration), Constraints on mediator-based dark matter and scalar dark energy models using $\sqrt{\mathrm{s}}=13 \mathrm{TeV}$ pp collision data collected by the ATLAS detector, J. High Energy Phys. 05 (2019) 142.

[11] D. S. Akerib et al. (LUX Collaboration), Results from a Search for Dark Matter in the Complete LUX Exposure, Phys. Rev. Lett. 118, 021303 (2017).

[12] X. Cui et al. (PandaX-II Collaboration), Dark Matter Results From 54-Ton-Day Exposure of PandaX-II Experiment, Phys. Rev. Lett. 119, 181302 (2017).

[13] E. Aprile et al. (XENON Collaboration), Dark Matter Search Results from a One Ton-Year Exposure of XENON1T, Phys. Rev. Lett. 121, 111302 (2018).

[14] P. Agnes et al. (DarkSide Collaboration), Low-Mass Dark Matter Search with the DarkSide-50 Experiment, Phys. Rev. Lett. 121, 081307 (2018).

[15] R. Agnese et al. (SuperCDMS Collaboration), First Dark Matter Constraints from a SuperCDMS Single-Charge Sensitive Detector, Phys. Rev. Lett. 121, 051301 (2018); Erratum, Phys. Rev. Lett. 122, 069901 (2019).

[16] E. Aprile et al. (XENON Collaboration), Constraining the Spin-Dependent WIMP-Nucleon Cross Sections with XENON1T, Phys. Rev. Lett. 122, 141301 (2019). 
[17] C. Amole et al. (PICO Collaboration), Dark matter search results from the complete exposure of the PICO-60 $\mathrm{C}_{3} \mathrm{~F}_{8}$ bubble chamber, Phys. Rev. D 100, 022001 (2019).

[18] G. Adhikari et al. (COSINE-100 Collaboration), Search for a Dark Matter-Induced Annual Modulation Signal in $\mathrm{NaI}(\mathrm{Tl})$ with the COSINE-100 Experiment, Phys. Rev. Lett. 123, 031302 (2019).

[19] R. Ajaj et al. (DEAP Collaboration), Search for dark matter with a 231-day exposure of liquid argon using DEAP-3600 at SNOLAB, Phys. Rev. D 100, 022004 (2019).

[20] A. H. Abdelhameed et al. (CRESST Collaboration), First results from the CRESST-III low-mass dark matter program, Phys. Rev. D 100, 102002 (2019).

[21] V. Barger, W.-Y. Keung, and G. Shaughnessy, Spin dependence of dark matter scattering, Phys. Rev. D 78, 056007 (2008).

[22] G. Belanger, F. Boudjema, A. Pukhov, and A. Semenov, Dark matter direct detection rate in a generic model with Micromegas 2.2, Comput. Phys. Commun. 180, 747 (2009).

[23] P. Agrawal, Z. Chacko, C. Kilic, and R. K. Mishra, A classification of dark matter candidates with primarily spin-dependent interactions with matter, arXiv:1003.1912.

[24] H. Goldberg, Constraint on the Photino Mass from Cosmology, Phys. Rev. Lett. 50, 1419 (1983); Erratum, Phys. Rev. Lett. 103, 099905 (2009).

[25] J. R. Ellis, J. S. Hagelin, D. V. Nanopoulos, K. A. Olive, and M. Srednicki, Supersymmetric Relics from the Big Bang, Nucl. Phys. B238, 453 (1984).

[26] M. Drees and M. M. Nojiri, The neutralino relic density in minimal $\mathrm{N}=1$ supergravity, Phys. Rev. D 47, 376 (1993).

[27] M. Drees and F. Hajkarim, Neutralino dark matter in scenarios with early matter domination, J. High Energy Phys. 12 (2018) 042.

[28] G. Bélanger, G. Drieu La Rochelle, B. Dumont, R. M. Godbole, S. Kraml, and S. Kulkarni, LHC constraints on light neutralino dark matter in the MSSM, Phys. Lett. B 726, 773 (2013).

[29] A. Choudhury and A. Datta, Neutralino dark matter confronted by the LHC constraints on Electroweak SUSY signals, J. High Energy Phys. 09 (2013) 119.

[30] T. Han, Z. Liu, and S. Su, Light neutralino dark matter: Direct/indirect detection and collider searches, J. High Energy Phys. 08 (2014) 093.

[31] N. Arkani-Hamed, A. Delgado, and G. Giudice, The Welltempered neutralino, Nucl. Phys. B741, 108 (2006).

[32] M. Chakraborti, U. Chattopadhyay, and S. Poddar, How light a higgsino or a wino dark matter can become in a compressed scenario of MSSM, J. High Energy Phys. 09 (2017) 064.

[33] M. Cahill-Rowley, R. Cotta, A. Drlica-Wagner, S. Funk, J. Hewett, A. Ismail, T. Rizzo, and M. Wood, Complementarity of dark matter searches in the phenomenological MSSM, Phys. Rev. D 91, 055011 (2015).

[34] M. Chakraborti, U. Chattopadhyay, S. Rao, and D. P. Roy, Higgsino dark matter in nonuniversal gaugino mass models, Phys. Rev. D 91, 035022 (2015).

[35] A. Delgado and M. Quirós, Higgsino dark matter in the MSSM, Phys. Rev. D 103, 015024 (2021).
[36] A. Pierce, Dark matter in the finely tuned minimal supersymmetric Standard Model, Phys. Rev. D 70, 075006 (2004).

[37] I. Gogoladze, R. Khalid, Y. Mimura, and Q. Shafi, Direct and indirect detection and LHC signals of Bino-Higgsino dark matter, Phys. Rev. D 83, 095007 (2011).

[38] M. van Beekveld, W. Beenakker, S. Caron, R. Peeters, and R. Ruiz de Austri, Supersymmetry with Dark Matter is still natural, Phys. Rev. D 96, 035015 (2017).

[39] M. Abdughani, L. Wu, and J. M. Yang, Status and prospects of light bino-higgsino dark matter in natural SUSY, Eur. Phys. J. C 78, 4 (2018).

[40] H. Baer, A. Mustafayev, E.-K. Park, and S. Profumo, Mixed wino dark matter: Consequences for direct, indirect and collider detection, J. High Energy Phys. 07 (2005) 046.

[41] H. Baer, T. Krupovnickas, A. Mustafayev, E.-K. Park, S. Profumo, and X. Tata, Exploring the BWCA (bino-wino co-annihilation) scenario for neutralino dark matter, J. High Energy Phys. 12 (2005) 011.

[42] A. Birkedal-Hansen and B. D. Nelson, The role of Wino content in neutralino dark matter, Phys. Rev. D 64, 015008 (2001).

[43] H. Baer, V. Barger, and H. Serce, SUSY under siege from direct and indirect WIMP detection experiments, Phys. Rev. D 94, 115019 (2016).

[44] M. Badziak, M. Olechowski, and P. Szczerbiak, Is welltempered neutralino in MSSM still alive after 2016 LUX results?, Phys. Lett. B 770, 226 (2017).

[45] S. Profumo, T. Stefaniak, and L. Stephenson Haskins, The not-so-well tempered neutralino, Phys. Rev. D 96, 055018 (2017).

[46] M. Guchait and A. Roy, Light Singlino Dark Matter at the LHC, Phys. Rev. D 102, 075023 (2020).

[47] W. Abdallah, A. Chatterjee, and A. Datta, Revisiting singlino dark matter of the natural $\mathrm{Z}_{3}$-symmetric NMSSM in the light of LHC, J. High Energy Phys. 09 (2019) 095.

[48] R. K. Barman, G. Bélanger, B. Bhattacherjee, R. Godbole, D. Sengupta, and X. Tata, Current bounds and future prospects of light neutralino dark matter in NMSSM, Phys. Rev. D 103, 015029 (2021).

[49] R. Kumar Barman, G. Belanger, and R. M. Godbole, Status of low mass LSP in SUSY, Eur. Phys. J. Special Topics 229, 3159 (2020).

[50] W. Abdallah, A. Datta, and S. Roy, A relatively light, highly bino-like dark matter in the $\mathrm{Z}_{3}$-symmetric NMSSM and recent LHC searches, J. High Energy Phys. 04 (2021) 122.

[51] C. Cheung, L. J. Hall, D. Pinner, and J. T. Ruderman, Prospects and blind spots for neutralino dark matter, J. High Energy Phys. 05 (2013) 100.

[52] C. Cheung and D. Sanford, Simplified models of mixed dark matter, J. Cosmol. Astropart. Phys. 02 (2014) 011.

[53] P. Huang and C. E. M. Wagner, Blind spots for neutralino Dark Matter in the MSSM with an intermediate $\mathrm{m}_{\mathrm{A}}$, Phys. Rev. D 90, 015018 (2014).

[54] T. Han, F. Kling, S. Su, and Y. Wu, Unblinding the dark matter blind spots, J. High Energy Phys. 02 (2017) 057.

[55] J. Cao, L. Meng, Y. Yue, H. Zhou, and P. Zhu, Suppressing the scattering of WIMP dark matter and nucleons in 
supersymmetric theories, Phys. Rev. D 101, 075003 (2020).

[56] R. Barbieri and G. F. Giudice, Upper bounds on supersymmetric particle masses, Nucl. Phys. B306, 63 (1988).

[57] L. Giusti, A. Romanino, and A. Strumia, Natural ranges of supersymmetric signals, Nucl. Phys. B550, 3 (1999).

[58] R. Kitano and Y. Nomura, Supersymmetry, naturalness, and signatures at the LHC, Phys. Rev. D 73, 095004 (2006).

[59] R. Barbieri and D. Pappadopulo, S-particles at their naturalness limits, J. High Energy Phys. 10 (2009) 061.

[60] M. Asano, H. D. Kim, R. Kitano, and Y. Shimizu, Natural supersymmetry at the LHC, J. High Energy Phys. 12 (2010) 019.

[61] H. Baer, V. Barger, P. Huang, D. Mickelson, A. Mustafayev, and $\mathrm{X}$. Tata, Radiative natural supersymmetry: Reconciling electroweak fine-tuning and the Higgs boson mass, Phys. Rev. D 87, 115028 (2013).

[62] T. Moroi and L. Randall, Wino cold dark matter from anomaly mediated SUSY breaking, Nucl. Phys. B570, 455 (2000).

[63] T. Gherghetta, G. F. Giudice, and J. D. Wells, Phenomenological consequences of supersymmetry with anomaly induced masses, Nucl. Phys. B559, 27 (1999).

[64] A. Arbey, A. Deandrea, and A. Tarhini, Anomaly mediated SUSY breaking scenarios in the light of cosmology and in the dark (matter), J. High Energy Phys. 05 (2011) 078.

[65] J. Hisano, K. Ishiwata, N. Nagata, and T. Takesako, Direct detection of electroweak-interacting dark matter, J. High Energy Phys. 07 (2011) 005.

[66] R. J. Hill and M. P. Solon, Universal behavior in the scattering of heavy, weakly interacting dark matter on nuclear targets, Phys. Lett. B 707, 539 (2012).

[67] G. Aad et al. (ATLAS Collaboration), Search for Heavy Higgs Bosons Decaying Into Two Tau Leptons with the ATLAS Detector Using pp Collisions at $\sqrt{\mathrm{s}}=13 \mathrm{TeV}$, Phys. Rev. Lett. 125, 051801 (2020).

[68] A. M. Sirunyan et al. (CMS Collaboration), Search for additional neutral MSSM Higgs bosons in the $\tau \tau$ final state in proton-proton collisions at $\sqrt{\mathrm{s}}=13 \mathrm{TeV}$, J. High Energy Phys. 09 (2018) 007.

[69] S. Gori, P. Schwaller, and C. E. M. Wagner, Search for Higgs bosons in SUSY cascade decays and neutralino dark matter, Phys. Rev. D 83, 115022 (2011).

[70] D. Ghosh, M. Guchait, and D. Sengupta, Higgs signal in chargino-neutralino production at the LHC, Eur. Phys. J. C 72, 2141 (2012).

[71] V. D. Barger, M. S. Berger, P. Ohmann, and R. J. N. Phillips, Multi-lepton SUSY signals from R-parity violation at the Tevatron, Phys. Rev. D 50, 4299 (1994).

[72] H. Baer, C.-h. Chen, F. Paige, and X. Tata, Trileptons from chargino-neutralino production at the CERN large hadron collider, Phys. Rev. D 50, 4508 (1994).

[73] B. Dutta, T. Kamon, N. Kolev, K. Sinha, K. Wang, and S. $\mathrm{Wu}$, Top squark searches using dilepton invariant mass distributions and Bino-Higgsino dark matter at the LHC, Phys. Rev. D 87, 095007 (2013).

[74] C. Han, Probing light bino and higgsinos at the LHC, Int. J. Mod. Phys. A 32, 1745003 (2017).
[75] M. van Beekveld, W. Beenakker, S. Caron, and R. Ruiz de Austri, The case for $100 \mathrm{GeV}$ bino dark matter: A dedicated LHC tri-lepton search, J. High Energy Phys. 04 (2016) 154.

[76] J. Liu, N. McGinnis, C. E. M. Wagner, and X.-P. Wang, Searching for the Higgsino-Bino sector at the LHC, J. High Energy Phys. 09 (2020) 073.

[77] A. Djouadi, J.-L. Kneur, and G. Moultaka, SuSpect: A Fortran code for the supersymmetric and Higgs particle spectrum in the MSSM, Comput. Phys. Commun. 176, 426 (2007).

[78] A. Djouadi, M. Muhlleitner, and M. Spira, Decays of supersymmetric particles: The Program SUSY-HIT (SUspect-SdecaY-Hdecay-InTerface), Acta Phys. Pol. B 38, 635 (2007), https://www.actaphys.uj.edu.pl/R/38/2/ $635 /$ pdf.

[79] G. Belanger, F. Boudjema, C. Hugonie, A. Pukhov, and A. Semenov, Relic density of dark matter in the NMSSM, J. Cosmol. Astropart. Phys. 09 (2005) 001.

[80] G. Belanger, F. Boudjema, A. Pukhov, and A. Semenov, MicromegAs: Version 1.3, Comput. Phys. Commun. 174, 577 (2006).

[81] G. Belanger, F. Boudjema, A. Pukhov, and A. Semenov, MicromEGAs 2.0: A Program to calculate the relic density of dark matter in a generic model, Comput. Phys. Commun. 176, 367 (2007).

[82] G. Belanger, F. Boudjema, A. Pukhov, and A. Semenov, MicrOMEGAs 3: A program for calculating dark matter observables, Comput. Phys. Commun. 185, 960 (2014).

[83] LEPSUSYWG, Aleph, Delphi, L3 and OPAL experiments, note LEPSUSYWG/01-03.1, Combined lep chargino mass limits. http://lepsusy.web.cern.ch/lepsusy.

[84] P. Bechtle, O. Brein, S. Heinemeyer, G. Weiglein, and K. E. Williams, HiggsBounds: Confronting arbitrary higgs sectors with exclusion bounds from LEP and the Tevatron, Comput. Phys. Commun. 181, 138 (2010).

[85] P. Bechtle, O. Brein, S. Heinemeyer, G. Weiglein, and K. E. Williams, HiggsBounds 2.0.0: Confronting neutral and charged higgs sector predictions with exclusion bounds from LEP and the Tevatron, Comput. Phys. Commun. 182, 2605 (2011).

[86] P. Bechtle, O. Brein, S. Heinemeyer, O. Stal, T. Stefaniak, G. Weiglein, and K. Williams, Recent developments in HiggsBounds and a preview of HiggsSignals, Proc. Sci., CHARGED2012 (2012) 024.

[87] P. Bechtle, O. Brein, S. Heinemeyer, O. Stål, T. Stefaniak, G. Weiglein, and K. E. Williams, HiggsBounds-4: Improved Tests of Extended Higgs Sectors against Exclusion Bounds from LEP, the Tevatron and the LHC, Eur. Phys. J. C 74, 2693 (2014).

[88] P. Bechtle, S. Heinemeyer, O. Stal, T. Stefaniak, and G. Weiglein, Applying Exclusion Likelihoods from LHC Searches to Extended Higgs Sectors, Eur. Phys. J. C 75, 421 (2015).

[89] A. M. Sirunyan et al. (CMS Collaboration), Search for supersymmetry in proton-proton collisions at $13 \mathrm{TeV}$ in final states with jets and missing transverse momentum, J. High Energy Phys. 10 (2019) 244.

[90] A. M. Sirunyan et al. (CMS Collaboration), Searches for physics beyond the standard model with the $\mathrm{M}_{\mathrm{T} 2}$ variable 
in hadronic final states with and without disappearing tracks in proton-proton collisions at $\sqrt{\mathrm{s}}=13 \mathrm{TeV}$, Eur. Phys. J. C 80, 3 (2020).

[91] A. M. Sirunyan et al. (CMS Collaboration), Search for direct top squark pair production in events with one lepton, jets, and missing transverse momentum at $13 \mathrm{TeV}$ with the CMS experiment, J. High Energy Phys. 05 (2020) 032.

[92] CMS Collaboration, Search for top squark pair production in the dilepton final state using $137 / \mathrm{fb}$ of proton-proton collision integrated luminosity at $\sqrt{\mathrm{s}}=13 \mathrm{TeV}$, Technical Report No. CMS-PAS-SUS-19-011, CERN, Geneva, 2020, https://cds.cern.ch/record/2718813.

[93] G. Aad et al. (ATLAS Collaboration), Search for a scalar partner of the top quark in the all-hadronic $\bar{t} \bar{t}$ plus missing transverse momentum final state at $\sqrt{\mathrm{s}}=13 \mathrm{TeV}$ with the ATLAS detector, Eur. Phys. J. C 80, 737 (2020).

[94] ATLAS Collaboration, Search for new phenomena with top quark pairs in final states with one lepton, jets, and missing transverse momentum in pp collisions at $\sqrt{\mathrm{s}}=$ $13 \mathrm{TeV}$ with the ATLAS detector, Technical Report No. ATLAS-CONF-2020-003, CERN, Geneva, 2020, https://cds.cern.ch/record/2711489.

[95] ATLAS Collaboration, Search for new phenomena in events with two opposite-charge leptons, jets and missing transverse momentum in $\mathrm{pp}$ collisions at $\sqrt{\mathrm{s}}=13 \mathrm{TeV}$ with the ATLAS detector, Technical Report No. ATLASCONF-2020-046, CERN, Geneva, 2020, https://cds.cern .ch/record/2728056.

[96] M. Aaboud et al. (ATLAS Collaboration), Search for topsquark pair production in final states with one lepton, jets, and missing transverse momentum using $36 \mathrm{fb}^{-1}$ of $\sqrt{\mathrm{s}}=$ $13 \mathrm{TeV}$ pp collision data with the ATLAS detector, J. High Energy Phys. 06 (2018) 108.

[97] S. Kraml, S. Kulkarni, U. Laa, A. Lessa, W. Magerl, D. Proschofsky-Spindler, and W. Waltenberger, sModels: A tool for interpreting simplified-model results from the LHC and its application to supersymmetry, Eur. Phys. J. C 74, 2868 (2014).

[98] F. Ambrogi, S. Kraml, S. Kulkarni, U. Laa, A. Lessa, V. Magerl, J. Sonneveld, M. Traub, and W. Waltenberger, SModelS v1.1 user manual: Improving simplified model constraints with efficiency maps, Comput. Phys. Commun. 227, 72 (2018).

[99] T. Sjostrand, S. Mrenna, and P.Z. Skands, PYTHIA 6.4 physics and manual, J. High Energy Phys. 05 (2006) 026.

[100] T. Sjostrand, S. Mrenna, and P.Z. Skands, A brief introduction to PYTHIA 8.1, Comput. Phys. Commun. 178, 852 (2008).

[101] J. Alwall, R. Frederix, S. Frixione, V. Hirschi, F. Maltoni, O. Mattelaer, H. S. Shao, T. Stelzer, P. Torrielli, and M. Zaro, The automated computation of tree-level and nextto-leading order differential cross sections, and their matching to parton shower simulations, J. High Energy Phys. 07 (2014) 079.

[102] P. Z. Skands et al., SUSY Les Houches accord: Interfacing SUSY spectrum calculators, decay packages, and event generators, J. High Energy Phys. 07 (2004) 036.
[103] J. de Favereau, C. Delaere, P. Demin, A. Giammanco, V. Lemaître, A. Mertens, and M. Selvaggi (DELPHES 3 Collaboration), DELPHES 3, A modular framework for fast simulation of a generic collider experiment, J. High Energy Phys. 02 (2014) 057.

[104] V. Khachatryan et al. (CMS Collaboration), Search for supersymmetry in pp collisions at $\sqrt{\mathrm{s}}=13 \mathrm{TeV}$ in the single-lepton final state using the sum of masses of largeradius jets, J. High Energy Phys. 08 (2016) 122.

[105] M. Cacciari, G. P. Salam, and G. Soyez, FAstJet user manual, Eur. Phys. J. C 72, 1896 (2012).

[106] Y. L. Dokshitzer, G. D. Leder, S. Moretti, and B. R. Webber, Better jet clustering algorithms, J. High Energy Phys. 08 (1997) 001.

[107] J. M. Butterworth, A. R. Davison, M. Rubin, and G. P. Salam, Jet Substructure as a New Higgs Search Channel at the LHC, Phys. Rev. Lett. 100, 242001 (2008).

[108] M. Dasgupta, A. Fregoso, S. Marzani, and G. P. Salam, Towards an understanding of jet substructure, J. High Energy Phys. 09 (2013) 029.

[109] M. Cacciari, G. P. Salam, and G. Soyez, The anti-k $\mathrm{k}_{\mathrm{t}}$ jet clustering algorithm, J. High Energy Phys. 04 (2008) 063.

[110] R. D. Ball, L. Del Debbio, S. Forte, A. Guffanti, J. I. Latorre, J. Rojo, and M. Ubiali, A first unbiased global NLO determination of parton distributions and their uncertainties, Nucl. Phys. B838, 136 (2010).

[111] A. Broggio, A. Ferroglia, M. Neubert, L. Vernazza, and L. L. Yang, Approximate NNLO predictions for the stoppair production cross section at the LHC, J. High Energy Phys. 07 (2013) 042.

[112] K. Melnikov and M. Schulze, NLO QCD corrections to top quark pair production and decay at hadron colliders, J. High Energy Phys. 08 (2009) 049.

[113] N. Kidonakis and R. Vogt, The theoretical top quark cross section at the Tevatron and the LHC, Phys. Rev. D 78, 074005 (2008).

[114] W. Beenakker, S. Dittmaier, M. Kramer, B. Plumper, M. Spira, and P. M. Zerwas, NLO QCD corrections to t anti-t $\mathrm{H}$ production in hadron collisions, Nucl. Phys. B653, 151 (2003).

[115] A. Kardos, Z. Trocsanyi, and C. Papadopoulos, Top quark pair production in association with a Z-boson at NLO accuracy, Phys. Rev. D 85, 054015 (2012).

[116] F. Buccioni, S. Kallweit, S. Pozzorini, and M. F. Zoller, NLO QCD predictions for $\bar{t} \bar{b} \bar{b}$ production in association with a light jet at the LHC, J. High Energy Phys. 12 (2019) 015 .

[117] A. Hocker et al., TMVA-Toolkit for multivariate data analysis, arXiv:physics/0703039.

[118] H. Voss, A. Hocker, J. Stelzer, and F. Tegenfeldt, TMVA, the toolkit for multivariate data analysis with ROOT, Proc. Sci., ACAT2007 (2007) 040.

[119] J. Friedman, T. Hastie, and R. Tibshirani, The Elements of Statistical Learning, Springer Series in Statistics (2001).

[120] A. Webb, Statistical Pattern Recognition, 2nd ed. (J. Wiley \& Sons Ltd, New York, 2002).

[121] L. Kuncheva, Combining Pattern Classifiers (J. Wiley \& Sons, New York, 2004). 